\title{
Physical parameters affecting sonoluminescence: A self-consistent hydrodynamic study
}

\author{
L. Yuan, ${ }^{1,2}$ H. Y. Cheng, ${ }^{1}$ M.-C. Chu, ${ }^{1}$ and P. T. Leung ${ }^{1}$ \\ ${ }^{1}$ Department of Physics, The Chinese University of Hong Kong, Shatin, New Territories, Hong Kong \\ ${ }^{2}$ LNM, Institute of Mechanics, Academia Sinica, Beijing 100080, People's Republic of China
}

(Received 7 October 1997)

\begin{abstract}
We studied the dependence of thermodynamic variables in a sonoluminescing (SL) bubble on various physical factors, which include viscosity, thermal conductivity, surface tension, the equation of state of the gas inside the bubble, as well as the compressibility of the surrounding liquid. The numerical solutions show that the existence of shock waves in the SL parameter regime is very sensitive to these factors. Furthermore, we show that even without shock waves, the reflection of continuous compressional waves at the bubble center can produce the high temperature and picosecond time scale light pulse of the SL bubble, which implies that SL may not necessarily be due to shock waves. [S1063-651X(98)09504-X]

PACS number(s): 78.60.Mq, 47.40.-x, 44.10.+i, 43.25.+y
\end{abstract}

\section{INTRODUCTION}

The phenomenon of sonoluminescence (SL) associated with the collapse of a gas bubble driven by ultrasound has aroused considerable interest due to recent experimental advances [1]. However, the phenomenon is not yet completely understood. A popular idea is that the enormous temperature and pressure within the bubble that cause sonoluminescence are produced by imploding shock waves that focus at the bubble center. These ideas were first put forward by Jarman [2] in the 1960s, but were put to numerical tests only recently by Wu and Roberts [3], Moss et al. [4], and Kondić et al. [5] after discarding the assumption of uniform pressure, temperature, and linear dependence of the velocity in radial coordinate in earlier works. However, these inviscid models did not take into account thermal conduction, surface tension, and viscosity. Furthermore, these works made use of a form of the Rayleigh-Plesset (RP) equation that includes effects of acoustic radiation but not corrections due to finite liquid compressibility.

The importance of surface tension, viscosity, heat conduction, and liquid compressibility to nonlinear oscillations of the bubble as well as sonoluminescence are more or less studied or mentioned in previous works. The role of the surface tension has been illustrated by Brenner et al. [6] and studied recently by Akhatov et al. [7]. Flynn [8] mentioned that viscosity and compressibility of the liquid always damp the bubble motions while heat conduction in general increases the violence of bubble motions. The critical importance of diffusive transport to sonoluminescence was underscored by many researchers. The effect of thermal conduction between the bubble and the surrounding liquid was studied by Hickling [9] by assuming a constant interface temperature. He found that for bubbles smaller than $10 \mu \mathrm{m}$ at ordinary conditions, the thermal diffusion length is about 10 $\mu \mathrm{m}$ in $0.5 \mu \mathrm{s}$, comparable to the dimensions of the bubble. Chu and Leung [10] reexamined the effects of thermal conduction and found that bubbles of several $\mu \mathrm{m}$ radii remain close to being isothermal during the expansion phase. Crum and Cordry [11], Kwak and Yang [12], Kamath et al. [13], and Yasui [14] also studied the effect of thermal conduction. They found that heat transfer into the bubble during the long, slow expansion phase of the oscillation plays a crucial role in determining the peak temperature within the bubble. However, in analyzing gas dynamics, simplifying assumptions such as linear velocity or uniform pressure distribution were made, which may break down at higher amplitudes of the acoustic forcing.

In a more recent work, Vuong and Szeri [15] developed a more realistic Navier-Stokes (NS) model for the gas dynamics within the bubble and studied the diffusive transport at large amplitudes of acoustic forcing. They demonstrated that there are no sharp shocks focusing at the origin of a noble gas bubble at sonoluminescing conditions and that energy focusing comes from wavy disturbances. However, they neglected surface tension and in effect overestimated the peak temperatures of the bubble.

No discussions were made up to now about the relative merit of various equations of state (EOS's). Most of the past studies used the van der Waals EOS considering the enormous compression that may occur. However, for air or noble gas-doped nitrogen bubbles, there may be dissociation and subsequent chemical reactions as temperature reaches a certain threshold. A realistic EOS should account for these effects. Moss et al. [4] used an EOS that takes into account vibrational excitation, dissociation, ionization, and a repulsive intermolecular potential. However, in using their model we found that it led to a minimum bubble radius comparable to that from an ideal gas EOS, but generally smaller than that observed experimentally [1], thus resulting in so high a temperature as can, they claimed, cause "microthermonuclear fusion." A hard-core potential seems to be essential to reproduce the correct minimum bubble radius. Another drawback of this EOS is its incapability to model low temperature, high density gas, which is the condition near the bubble wall. We are also not aware of any systematic study of the effects of the liquid compressibility, even though this effect is clearly important in a short stage of bubble collapse.

In what follows we shall present a self-consistent hydrodynamic model of the bubble oscillation that includes the 
effects of liquid compressibility, thermal conduction, viscosity, surface tension, the bubble content, and the equation of states. Incorporating all these potentially important effects in a reliable hydrodynamic calculation allows us to study the effects of each physical parameter on sonoluminescence. Our major conclusion is that while shock waves are formed in some parameter regimes, their strength, and indeed their existence, are highly sensitive to liquid compressibility, thermal conduction, surface tension, and the equation of state. Shocks are not robust in sonoluminescing bubbles. However, compressional waves emerge naturally from the hydrodynamics regardless of the existence of shock waves, and they give rise to temperature and power pulse widths comparable to experimental numbers.

\section{HYDRODYNAMIC MODELS}

We shall assume the bubble motion to be spherically symmetric and ignore mass diffusion as previous works did. It is not clear yet whether a sonoluminescing bubble remains spherically symmetric throughout its oscillation. Nevertheless, we focus our study on spherically symmetric hydrodynamics, so that the roles of other physical parameters can be revealed without being burdened with intensive computation. Mass diffusion determines the ambient radius of the bubble, the variation of the gas species, and above all the stability of the bubble. However, for a stable sonoluminescing bubble in water, the mass of gas transported into and out of the bubble are equal and only accounts for about $1 \%$ of the total mass in the bubble [17]. The time scale associated with mass diffusion is also long compared with the period of oscillation, and so we ignore this process in our calculations. We also ignore the effects of light and heat radiation on the hydrodynamics. These may become important only at high temperature and result in little loss of energy throughout most of an oscillation period. Inclusion of these will favor our conclusion about the weakening of shock waves even more. We do not intend to simulate the entire motion of the surrounding liquid as a compressible flow. Instead, the bubble wall motion is determined by the RP equation under near-incompressible assumption, and the energy equation for the liquid is solved independently. This avoids the difficult numerical task of simultaneously solving the mass, momentum and energy conservation equations for a nearly incompressible liquid.

Therefore, our hydrodynamic models consist of the Euler or Navier-Stokes equations and an EOS governing the motion of the gas inside the bubble, the RP equation governing the motion of the bubble radius as a moving boundary for the gas dynamics, and the energy balance equation in the liquid. In the following we first present these equations and the corresponding boundary conditions, and then we transform them into convenient forms and outline a Eulerian numerical method incorporating a total variation diminishing (TVD) scheme to solve the gas dynamics equations. The standard TVD scheme for the transformed gas equations is given in Appendix A.

\section{A. The RP equation for the bubble wall radius}

The bubble radius $R(t)$ obeys the RP equation. The first RP equation (RP1) we adopted was due to Rayleigh, Plesset, Noltingk, and Prostsky, for an incompressible flow and modified by Keller and Kolodner to include acoustic radiation [16]. It can be written as $[6,17]$

$$
\begin{aligned}
R \ddot{R}+\frac{3}{2} \dot{R}^{2}= & \frac{1}{\rho_{l_{\infty}}}\left[P_{g}(R, t)-\frac{2 \sigma}{R}-\frac{4 \eta \dot{R}}{R}-P_{\infty}-P_{s}(t)\right] \\
& +\frac{t_{R}}{\rho_{l \infty}} \frac{d}{d t}\left[P_{g}(R, t)-P_{s}(t)\right] .
\end{aligned}
$$

Here overdots denote time derivatives, $\rho_{l \infty}$ is the ambient liquid density, $P_{g}(R, t)$ the gas pressure, $P_{\infty}$ the ambient pressure, $P_{s}(t)=-P_{a} \sin (\omega t)$ the pressure of the sound field with frequency $\omega$ and amplitude $P_{a}, t_{R} \equiv R / c_{100}, c_{l 00}$ the speed of sound in the liquid at room temperature and pressure at $1 \mathrm{~atm}, \sigma$ the surface tension, and $\eta$ the dynamic viscosity of the liquid. The left-hand side of this equation represents the inertia of the accelerating bubble in response to the net force on it, which as written on the right-hand side, is due to the pressure difference across the bubble wall. Obviously, the viscous effects and acoustic radiation term in the right-hand side will damp the bubble's motion. The pressure $P_{g}(R, t)$ on the gas side of the bubble wall exceeds the pressure $P_{b}(t)$ on the liquid side of the bubble wall by the effect of the surface tension and the normal component of viscous stresses:

$$
P_{g}(R, t)-\left.\tau_{r r}\right|_{r=R}=P_{b}(t)+\frac{4 \eta \dot{R}}{R}+\frac{2 \sigma}{R},
$$

where $\tau_{r r}$ is the normal viscous stress of the gas. The gas pressure $P_{g}(R, t)$ should in general be determined from a consideration of the conservation equations inside the bubble, which will be described in the next subsection. Vapor pressure is ignored because its effect is small [13]. The second RP equation (RP2) follows from the Keller-Miksis formulation [19], which includes the effects of liquid compressibility:

$$
\begin{aligned}
(1-M) R \ddot{R}+\frac{3}{2}\left(1-\frac{M}{3}\right) \dot{R}^{2}= & \frac{1}{\rho_{l \infty}}(1+M)\left[P_{b}(t)-P_{\infty}\right. \\
& \left.-P_{s}\left(t+t_{R}\right)\right]+\frac{t_{R}}{\rho_{l \infty}} \frac{d P_{b}(t)}{d t} .
\end{aligned}
$$

This equation contains terms that depend on the bubble-wall Mach number $M \equiv \dot{R} / c_{l \infty}$, which characterizes the liquid compressibility. RP2 was shown to belong to a oneparameter family of approximate equations for the bubble radius that are formally first-order accurate in the bubblewall Mach number [18]. If one approximately sets $P_{s}(t$ $\left.+t_{R}\right) \approx P_{s}(t)+t_{R} d P_{s} / d t$ and let $M \rightarrow 0$ while retaining the acoustic radiation term, Eq. (3) reduces to Eq. (1). An equation close to the Keller form, but written in terms of the enthalpy of the liquid at the bubble wall rather than the pressure was recommended in Ref. [17]. The recommended form is similar to that from the Gilmore formulation [20] except that a term in $c_{l \infty}^{-2}$ is dropped, but acoustic radiation is included. However, the assumption of a constant speed of sound in the liquid often leads to violation of small bubblewall Mach number assumption on which the RP equation is 
based, because at large amplitudes of acoustic forcing associated with sonoluminescence, the bubble wall velocity may exceed the ambient speed of sound in the liquid. However, one can expect that before this extreme circumstance of bubble motions occurs, the speed of sound $c_{l}$ will increase as the liquid pressure increases. This will reduce $M$ so that the RP equation recovers its validity at least to some extent. To model the liquid compressibility more accurately, we let the speed of sound depend on the EOS of the liquid as done in the original Gilmore form. The modified Keller equation (RP3) is as follows [21]:

$$
\begin{aligned}
(1-M) R \ddot{R}+\frac{3}{2}\left(1-\frac{M}{3}\right) \dot{R}^{2}= & (1+M)\left[H_{b}-\frac{1}{\rho_{l}} P_{s}\left(t+t_{R}\right)\right] \\
& +t_{R} \frac{d}{d t} H_{b}
\end{aligned}
$$

where $\rho_{l}, c_{l}$ and $H_{b}$ are the density, speed of sound, and enthalpy of the liquid:

$$
c_{l}^{2} \equiv \frac{d P}{d \rho_{l}}, \quad H_{b} \equiv \int_{P_{\infty}}^{P} \frac{d P}{\rho_{l}} .
$$

It is clear that Eq. (4) reduces to Eq. (3) if $H_{b} \approx\left(P_{b}\right.$ $\left.-P_{\infty}\right) / \rho_{l \infty}$ and $c_{l}=c_{l \infty 0}$. For water, explicit expressions for $c_{l}$ and $H_{b}$ are obtained by using an EOS of the modified Tait form [18]

$$
\frac{P+B}{P_{\infty}+B}=\left(\frac{\rho_{l}}{\rho_{l \infty}}\right)^{n},
$$

where $B=3049.13$ bars, $n=7.15$ are valid for water up to $10^{5}$ bars. With Eqs. (5) and (6) we readily find

$$
c_{l}^{2}=\frac{n(P+B)}{\rho_{l}}, \quad H_{b}=\frac{n}{n-1}\left(\frac{P+B}{\rho_{l}}-\frac{P_{\infty}+B}{\rho_{l \infty}}\right) .
$$

\section{B. Conservation equations for the gas in the bubble}

In the presence of viscosity and heat conduction, the equations for the conservation of mass, momentum, and energy for the gas flow in spherical symmetry can be rewritten into conservative form with source terms [15]:

$$
\begin{gathered}
\frac{\partial \rho}{\partial t}+\frac{\partial}{\partial r}(\rho v)=-\frac{2 \rho v}{r} \\
\frac{\partial(\rho v)}{\partial t}+\frac{\partial}{\partial r}\left(\rho v^{2}+P\right)=-\frac{2 \rho v^{2}}{r}+\frac{1}{r^{2}} \frac{\partial}{\partial r}\left(r^{2} \tau_{r r}\right)+\frac{\tau_{r r}}{r} \\
\frac{\partial E}{\partial t}+\frac{\partial}{\partial r}(E+P) v= \\
+\frac{1}{r^{2}} \frac{\partial}{\partial r}\left[r^{2}\left(v \tau_{r r}+k \frac{\partial T}{\partial r}\right)\right]
\end{gathered}
$$

Here $E=\rho\left(e+v^{2} / 2\right)$ is the total energy per unit volume, $\rho$, $v, P, T, k$, and $e$ are the density, radial velocity, pressure, temperature, coefficient of thermal conductivity, and internal energy per unit mass, respectively. The normal viscous stress $\tau_{r r}$ is

$$
\tau_{r r}=\frac{4 \mu}{3}\left(\frac{\partial v}{\partial r}-\frac{v}{r}\right),
$$

where $\mu$ is the dynamic viscosity of gas. The viscous term $\tau_{r r}$ is present only in the Navier-Stokes equations, and it is ignored in the Euler equations.

Two kinds of EOS are used to model the gas inside the bubble. Firstly, a hard-core van der Waals EOS (VEOS) is chosen for both air and noble gas because it has been shown to be better than an ideal gas due to the enormous compression in the bubble [17]:

$$
P=R_{g} \frac{\rho T}{1-b \rho}, \quad e=c_{v} T=\frac{1-b \rho}{\gamma-1}\left(\frac{P}{\rho}\right) .
$$

Here $R_{g}=K / \widetilde{M}$ is the gas constant, with $K=8314 \mathrm{~m}^{2} / \mathrm{K} \mathrm{s}^{2}$, $\widetilde{M}$ the molecular mass, $c_{v}=R_{g} /(\gamma-1)$ the specific heat at constant volume, $\gamma$ the ratio of specific heats, and $b$ the van der Waals excluded volume.

Secondly, an EOS due to Moss et al. [4] (MEOS), which includes vibrational excitation, dissociation, ionization, and a repulsive intermolecular potential is also used for an air bubble:

$$
\begin{gathered}
P=R_{g} \rho T\left[1+m_{D}(1+2 m)\right] \\
+\frac{E_{c} \rho_{0}}{1-(3 / n)}\left[\left(\rho / \rho_{0}\right)^{(n / 3)+1}-\left(\rho / \rho_{0}\right)^{2}\right], \\
e=R_{g}\left[5 T / 2+\Theta /\left(e^{\Theta / T}-1\right)\right]\left(1-m_{D}\right)+m_{D} R_{g} T_{D} \\
+\frac{3}{2} R_{g} T\left(2 m_{D}\right)(1+m)+2 m_{D} R_{g} \sum_{i} m_{i} T_{i} \\
+E_{c} /(n / 3-1)\left[\left(\rho / \rho_{0}\right)^{n / 3}-\left(n \rho / 3 \rho_{0}\right)\right]+E_{c},
\end{gathered}
$$

where

$$
\begin{gathered}
m_{k}=0.5\left\{\tanh \left[7\left(T-0.9 T_{k}\right) / T_{k}\right]+\tanh (6.3)\right\}, \\
m=\sum_{i=1}^{5} m_{i}, \\
T_{1-5}=14.5,29.6,47.4,77.5, \\
\text { and } 97.5 \mathrm{eV} ; \quad T_{D}=9.7 \mathrm{eV}, \\
n \approx 9, \quad \rho_{0}=1113 \mathrm{~kg} \mathrm{~m}^{-3}, \\
E_{c}=2.52 \times 10^{5} \mathrm{~J} \mathrm{~kg}^{-1}, \Theta=3340 \mathrm{~K} .
\end{gathered}
$$

The hydrodynamic equations are supplemented by boundary conditions. At the center of the bubble there are several relationships that hold true as a consequence of the coordinate singularity. For viscous flow $v=0, \partial E / \partial r=0, \partial \rho / \partial r$ $=0$. For inviscid flow, the above formulae also hold true except when a shock wave focuses. A proper singularity treatment should use the similarity solution for the corre- 
sponding EOS instead of the general algorithm for Eqs. (8)(10) and is a troublesome task. For our purpose of predicting whether there will be shock waves and estimating their relative strength in terms of thermal properties in the inviscid calculations, the above boundary conditions seem a plausible numerical approximation to an exact inviscid solution with shock focusing, if the mesh is fine enough near the center.

The boundary conditions on the bubble wall are $v=\dot{R}$, and an adiabatic condition $\partial T / \partial r=0$ for the Euler equations. For the NS equations, both temperature and heat flux are required to be continuous across the bubble (see the next subsection). In addition, mass conservation of gas inside the bubble gives a condition for the gas density at the bubble wall.

\section{Energy equation in the liquid}

While accounting for the temperature changes in the liquid, we shall follow previous works and assume that the liquid compressibility and viscosity do not affect the heat transfer process. The temperature dependence of the transport properties is negligible as the variation of the liquid temperature is small. The energy equation in the liquid is [15]

$$
\frac{\partial T_{l}}{\partial t}+v_{l} \frac{\partial T_{l}}{\partial r}=\frac{k_{l}}{\rho_{l} c_{p, l}} \frac{1}{r^{2}} \frac{\partial}{\partial r}\left(r^{2} \frac{\partial T_{l}}{\partial r}\right),
$$

where $T_{l}$ is the temperature, $k_{l}$ is the thermal conductivity, and $c_{p, l}$ is the specific heat of the liquid. With the transformation of Plesset and Zwick [22] $h=\left[r^{3}-R^{3}(t)\right] / 3$ and $v_{l}$ $=R^{2} \dot{R} / r^{2}$, the above convection-diffusion equation simplifies to a diffusion equation:

$$
\frac{\partial T_{l}}{\partial t}=D_{l} \frac{\partial}{\partial h}\left[\left[3 h+R^{3}(t)\right]^{4 / 3} \frac{\partial T_{l}}{\partial h}\right],
$$

where $D_{l}=k_{l} / \rho_{l} c_{p, l}$ is the thermal diffusion coefficient. Following Grosh and Orszag [23], we made a nonlinear map to transform the semi-infinite domain $h \subset[0, \infty]$ to a finite computational domain $z \subset[0,1]$ by using $h=-R_{u}^{3} \ln (1-z)$. The resulting equation is [24]

$\frac{\partial}{\partial t}\left(\frac{R_{u}^{3}}{1-z} T_{l}\right)=D_{l} \frac{\partial}{\partial z}\left\{\left[R^{3}-3 R_{u}^{3} \ln (1-z)\right]^{4 / 3} \frac{(1-z)}{R_{u}^{3}} \frac{\partial T_{l}}{\partial z}\right\}$,

where $R_{u} \approx R_{\max }$ is a geometric parameter. The continuity of both the temperature and heat flux at the bubble wall implies

$$
\left.T_{l}(z, t)\right|_{z=0}=\left.T(r, t)\right|_{r=R},\left.\quad k_{l} \frac{\partial T_{l}}{\partial r}\right|_{z=0}=\left.k \frac{\partial T}{\partial r}\right|_{r=R}
$$

In the far-field, the liquid temperature is just the ambient temperature $T_{\infty}$ :

$$
\left.T_{l}(z, t)\right|_{z=1}=T_{\infty}
$$

\section{Power radiated by the bubble}

Assuming a blackbody model [4,27], we have the relation between the power radiated by the bubble and the temperature:

$$
\dot{E}(t)=\int_{0}^{R} 16 \pi \sigma_{s} \kappa \rho(r, t) T^{4}(r, t) r^{2} d r
$$

where $\sigma_{s}=5.68 \times 10^{-8} \mathrm{~W} \mathrm{~s}^{-1} \mathrm{~K}^{-2}$ is the Stefan-Boltzmann constant, and $\kappa \approx 3500 \mathrm{~cm}^{2} \mathrm{~g}^{-1}$ is the Planck opacity.

\section{NUMERICAL METHODS}

Before we solve the whole set of governing equations, i.e., Eqs. (8), (9), (10) with one EOS, one of Eqs. (1), (3), (4), and Eq. (17) under appropriate boundary conditions, we use $x \equiv r / R(t)$ to transform the gas dynamics equations Eqs. $(8),(9)$, and (10) into a form in a fixed coordinate $x \subset[0,1]$. The resulting equations are

$$
\begin{aligned}
& \frac{\partial(\rho R)}{\partial t}+\frac{\partial}{\partial x}[\rho v-\rho x \dot{R}]=-\frac{2 \rho v}{x}, \\
& \frac{\partial(\rho v R)}{\partial t}+\frac{\partial}{\partial x}\left[\left(\rho v^{2}+P\right)-\rho v x \dot{R}\right] \\
& =-\frac{2 \rho v^{2}}{x}+\frac{1}{x^{2}} \frac{\partial}{\partial x}\left(x^{2} \tau_{r r}\right)+\frac{\tau_{r r}}{x}, \\
& \frac{\partial(E R)}{\partial t}+\frac{\partial}{\partial x}[(E+P) v-E x \dot{R}] \\
& =-\frac{2 v(E+P)}{x} \\
& +\frac{1}{x^{2}} \frac{\partial}{\partial x}\left[x^{2}\left(v \tau_{r r}+\frac{k}{R} \frac{\partial T}{\partial x}\right)\right] .
\end{aligned}
$$

To apply a TVD scheme, we rewrite Eqs. (21)-(23) to a vector form:

$$
\frac{\partial \mathbf{Q}}{\partial t}+\frac{\partial \mathbf{F}(\mathbf{Q})}{\partial x}=\mathbf{S}(\mathbf{Q})+\mathbf{S}_{v}(\mathbf{Q})
$$

Where $\mathbf{Q}=R \mathbf{U}=R(\rho, \rho v, E)^{T}$ and the inviscid flux $\mathbf{F}$ is similar to that in one-dimensional (1D) Cartesian coordinate except for a moving grid term $-x \dot{R} \mathbf{U}$. Its Jacobian is $\hat{\mathbf{A}}=(\mathbf{A}$ $-x \dot{R} \mathbf{I}) / R$, where $\mathbf{A}$ is the same Jacobian as in 1D Cartesian coordinate. The application of second-order TVD schemes [25] to Eq. (24) is thus straightforward. We treat the source terms due to spherical coordinate and viscous and heat conduction terms explicitly. The time discretization is a secondorder predictor-corrector method for both the gas dynamics and the RP equation. If the same time advance method is applied to the energy equation Eq. (17), which is a diffusion equation, it will be unstable even with intolerably small time step. Therefore we use the implicit Crank-Nicolson time discretization to solve Eq. (17) to avoid numerical instabilities. A variable time step is used based on the Courant number to guarantee adequate temporal resolution. The calculations are 
performed with 400 points in the gas bubble and 50 points in the liquid. Because the TVD scheme we adopted is a standard one, we give it in Appendix A.

\section{NUMERICAL RESULTS}

We calculated the motion of both air bubble and argon bubble for one period of the acoustic forcing starting from an equilibrium radius $R_{0}$. In all cases, $R_{0}=4.5 \mu \mathrm{m}, P_{\infty}$ $=101325 \mathrm{~Pa}, \quad T_{\infty}=300 \mathrm{~K}, \quad \omega / 2 \pi=26.4 \mathrm{kHz}, \quad \eta=$ $10^{-3} \mathrm{~kg} \mathrm{~m}^{-1} \mathrm{~s}^{-1}, c_{l \infty}=1481 \mathrm{~m} \mathrm{~s}^{-1}, \rho_{l \infty}=1000 \mathrm{~kg} \mathrm{~m}^{-3}, \sigma$ $=0.0725 \mathrm{~kg} \mathrm{~s}^{-2}, \quad k_{l}=0.0609 \mathrm{~W} \mathrm{~m}^{-1} \mathrm{~K}^{-1}, \quad$ and $c_{p, l}=$ $4179 \mathrm{~J} \mathrm{~kg}^{-1} \mathrm{~K}^{-1}$.

We computed with two driving pressures, $P_{a}$ $=1.275 \mathrm{~atm}$ and $1.35 \mathrm{~atm}$ for an air bubble. The parameters for air are $\gamma=1.4$, molecular mass $\widetilde{M}=28.8 \mathrm{~g} \mathrm{~mole}^{-1}$, and the van der Waals excluded volume $b=1 / \rho_{\max }$ $=0.036 \ell \mathrm{mol}^{-1}$. The viscosity is given by Sutherland's law [26] as $\mu=\mu_{\infty}(T / 288.15)^{1.5} 398.55 /(T+110.4)$, where $\mu_{\infty}$ $=1.7894 \times 10^{-5} \mathrm{~kg} \mathrm{~m}^{-1} \mathrm{~s}^{-1}$, and the heat conductivity is assumed to be linear in $T$ [21], $k(T)=A T+B$ with $A=5.528 \times 10^{-5} \mathrm{~W} \mathrm{~m}^{-1} \mathrm{~K}^{-2}$ and $B=1.165 \times 10^{-2} \mathrm{~W}$ $\mathrm{m}^{-1} \mathrm{~K}^{-1}$.

For an argon bubble, only $P_{a}=1.3 \mathrm{~atm}$ was computed. The parameters are taken from Ref. [15], which gives $\gamma$ $=5 / 3, \widetilde{M}=39.948 \mathrm{~g} \mathrm{~mol}^{-1}$, the van der Waals excluded volume $\quad b=0.03219 \ell \mathrm{mol}^{-1}, \quad \mu(T)=\mu_{\infty} T / T_{\infty}, \quad$ where $\mu_{\infty}=2.25793 \times 10^{-5} \mathrm{~kg} \mathrm{~m} \mathrm{~s}^{-1}, \quad k(T)=k_{\infty} T / T_{\infty}$ with $k_{\infty}$ $=0.01764 \mathrm{~W} \mathrm{~m}^{-1} \mathrm{~K}^{-1}$.

In what follows, except where mentioned otherwise, we will use both the Euler and the Navier-Stokes equations coupled to the three forms of the RP equation, which include surface tension and viscosity. We calculate the energy equation in the liquid when solving the NS equations. A van der Waals EOS is adopted in all cases. Usage of MEOS for air bubble and zero surface tension in the RP equation for argon bubble will be mentioned in the corresponding discussion.

In the following, we shall discuss the effects of liquid compressibility, thermal conduction, the equation of state, surface tension, and the bubble contents on the thermodynamics and hydrodynamics of sonoluminescence. We shall also discuss the relation of picosecond pulses to shock waves.

\section{A. Liquid compressibility}

The effect of liquid compressibility is characterized by the Mach number $M=v / c_{l}$ in the RP equation. The first form of the RP equation (RP1) is widely used [3,5,15]. But as Prosperetti and Lezzi [18] have shown, even though it includes acoustic radiation, it is only of zeroth order accurate in the bubble-wall Mach number. The second form (RP2), known as the Keller formulation [19], and the third form (RP3) $[18,21]$, which is similar to the Gilmore formulation [20], both contain terms that are first order approximations to the liquid compressibility. We select the third form motivated by the conclusion of [18] that an equation similar to the Keller form but written in terms of the enthalpy of the liquid at the bubble wall rather than the pressure is better than the Keller form. In addition, a variable speed of sound in the liquid, as in the original Gilmore form, is more physical, especially

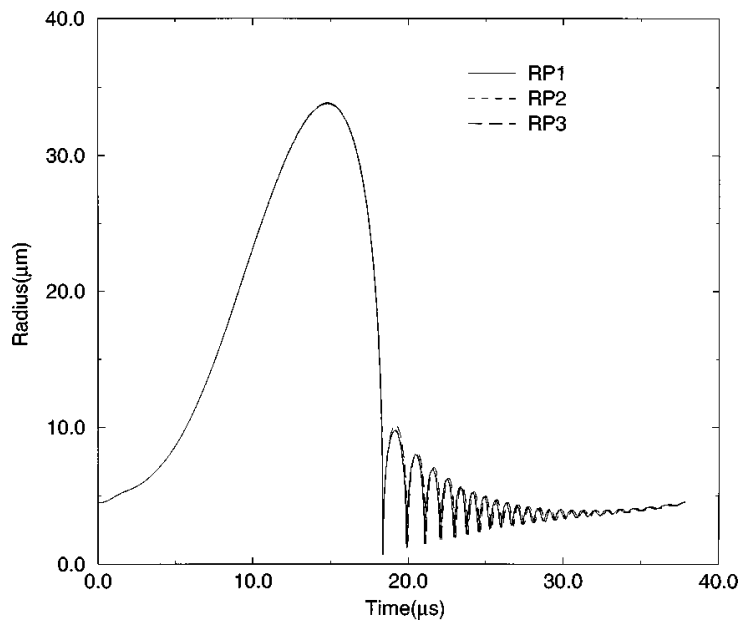

FIG. 1. Comparison of air bubble radius vs time in one period with various RP equations, for $R_{0}=4.5 \mu \mathrm{m}, f=26.4 \mathrm{kHz}, \sigma$ $=0.0725 \mathrm{~kg} \mathrm{~s}^{-2}$, and $P_{a}=1.275 \mathrm{~atm}$. The bubble interior is assumed to be uniform and stays at constant temperature in the expansion phase; adiabatic compression is assumed when the bubble radius is less than $R_{0}$.

during the violent collapse of the bubble. Initial comparisons among the three forms of RP equations were made in $[18,19]$ without coupling to gas dynamics within the bubble, and the latter two forms were shown to be more accurate.

We first study air bubbles driven at $P_{a}=1.275 \mathrm{~atm}$, which lies in the SL parameter regime and see how liquid compressibility affects the interface motion. The $R(t)$ curves as computed by the three RP equations alone are shown in Fig. 1. We can see that they are nearly the same before collapse, but there is a slight difference between RP3 and the former two RP equations after collapse. The effect of liquid compressibility on the $R(t)$ curve is almost negligible as $M$ is very small for most of the period but becomes significant in the very brief stage of collapse.

Now we couple the RP equations with the hydrodynamic equations inside and investigate how liquid compressibility affects the interface motion and the gas dynamics inside the bubble. Because of the highly nonlinear nature of the coupled system at larger $P_{a}$, one may expect that during the very brief stage of bubble collapse, of typically several hundred picoseconds long, the interface velocity becomes so large that the liquid compressibility plays an important role. The collapsing strength indeed depends sensitively on the liquid compressibility. In Table I, some extreme characteristics related to the bubble collapse are given. The last row is the result using MEOS to be discussed later in Sec. IV C. We see that the maximum bubble-wall velocities are all supersonic with respect to the ambient sound speed of air and may differ by about $20 \%-60 \%$. The bubble-wall velocity and thermal peaks of RP2 and RP3 are much smaller than those of RP1. This suggests that the liquid compressibility taken into account in RP2 and RP3 damps the bubble wall motion. As to why the collapsing strength of RP2 and RP3 is weaker, we suggest that the compressible liquid absorbs part of the incident acoustic energy and radiates more acoustic energy during the bubble's collapse. The compressible liquid surrounding the bubble acts like a "spring," preventing the 
TABLE I. Extrema values for air bubble collapse, $R_{0}=4.5 \mu \mathrm{m}, P_{a}=1.275 \mathrm{~atm}, f=26.4 \mathrm{kHz}$.

\begin{tabular}{lccccccc}
\hline \hline Gas dynamics & $\begin{array}{c}\text { RP } \\
\text { form }\end{array}$ & $\begin{array}{c}T_{\max } \\
\left(10^{3} \mathrm{~K}\right)\end{array}$ & $\begin{array}{c}P_{\max } \\
\left(10^{6} \mathrm{~atm}\right)\end{array}$ & $\begin{array}{c}\dot{R}_{\max } \\
\left(\mathrm{km} \mathrm{s}^{-1}\right)\end{array}$ & $\begin{array}{c}R_{\min } \\
(\mu \mathrm{m})\end{array}$ & $\begin{array}{c}R_{\max } \\
(\mu \mathrm{m})\end{array}$ & Shock wave \\
\hline \multirow{3}{*}{ Euler (adiabatic) } & $\mathrm{RP1}$ & 569 & 102 & 1.19 & 0.617 & 29.73 & Yes \\
& $\mathrm{RP2}$ & 5.37 & 0.03 & 0.87 & 0.634 & 29.88 & No \\
& $\mathrm{RP3}$ & 6.42 & 0.060 & 0.91 & 0.609 & 29.88 & No \\
NS & $\mathrm{RP1}$ & 315 & 27.7 & 1.64 & 0.597 & 35.43 & Yes \\
& $\mathrm{RP2}$ & 12.1 & .054 & 1.08 & 0.632 & 35.42 & No \\
NS (MEOS) & $\mathrm{RP3}$ & 15.0 & .116 & 1.20 & 0.593 & 35.47 & No \\
\hline \hline
\end{tabular}

bubble from a more violent collapse like that produced by RP1.

The effect of the liquid compressibility on shock formation can be seen clearly from the spatial profiles of various gas variables at several instances near $R_{\text {min }}$. In the Euler-RP1 result shown in Fig. 2 and Fig. 3, a shock wave appears at $t_{3}$ to $t_{4}$ and from $t_{7}$ to $t_{11}$, whereas the Euler-RP2 result (Fig. 4) and Euler-RP3 (not shown but similar to Fig. 4) contain no shocks although the variables are nonuniform in the bubble. The NS-RP1 solution also contains shock waves (Fig. 5 and Fig. 6) and focuses at $t_{8}$ in Fig. 6 while the NS-RP3 solution contains no shock as shown in Fig. 7.

At $P_{a}=1.35$ atm, our results show that the collapse motion of the bubble wall will become supersonic both in air and in water if a constant speed of sound $c_{l \infty}$ is used. The assumption on which the RP equations are based is no longer valid. However, we note that under such extreme conditions the liquid pressure adjacent to the bubble wall is also large ( $P_{b}=16855$ atm when $\dot{R}_{\max }=1740 \mathrm{~m} \mathrm{~s}^{-1}$ for the NS-RP3 solution), and a thin layer of liquid adjacent to the bubble is expected to be compressed so densely that the speed of sound there becomes larger according to Eqs. (6) and (7). Our calculation shows that the bubble-wall Mach number $M$ is always below 0.75 with RP3, thus making RP3 still a fair approximation.

The peak values are given in Table II for $P_{a}=1.35 \mathrm{~atm}$. All solutions have shock waves except the NS-RP3 solution with VEOS. RP1 gives extremely high temperature, pressure, and a larger bubble-wall velocity. The shock solutions computed by RP1 and RP2 are questionable because the bubble-wall Mach number exceeds 1, making the RP1 and RP2 invalid. The thermal peaks in the NS-RP3 model are larger than the corresponding $P_{a}=1.275$ atm values as expected. In Fig. 8 the spatial profiles of gas variables at sev-
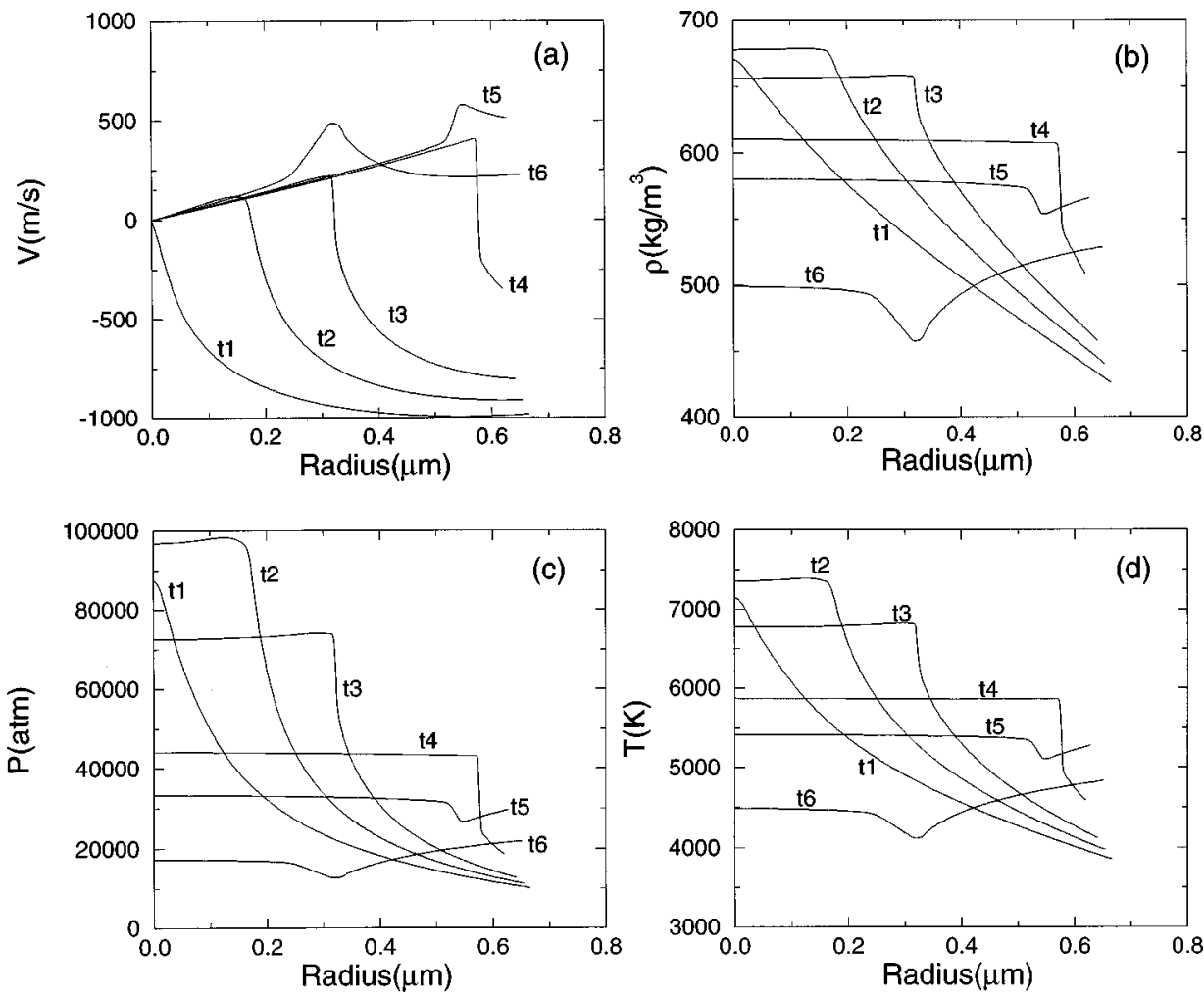

FIG. 2. Spatial profiles of (a) velocity, (b) density, (c) pressure, and (d) temperature, for an air bubble calculated with $R_{0}=4.5 \mu \mathrm{m}$, $f=26.4 \mathrm{kHz}, \sigma=0.0725 \mathrm{~kg} \mathrm{~s}^{-2}$, and $P_{a}=1.275 \mathrm{~atm}$, using Euler-RP1. Snapshots are shown at $t_{1}=19.264815 \mu \mathrm{s}, t_{2}=19.264828 \mu \mathrm{s}$, $t_{3}=19.264843 \mu \mathrm{s}, t_{4}=19.264879 \mu \mathrm{s}, t_{5}=19.264904 \mu \mathrm{s}$, and $t_{6}=19.264972 \mu \mathrm{s}$. 

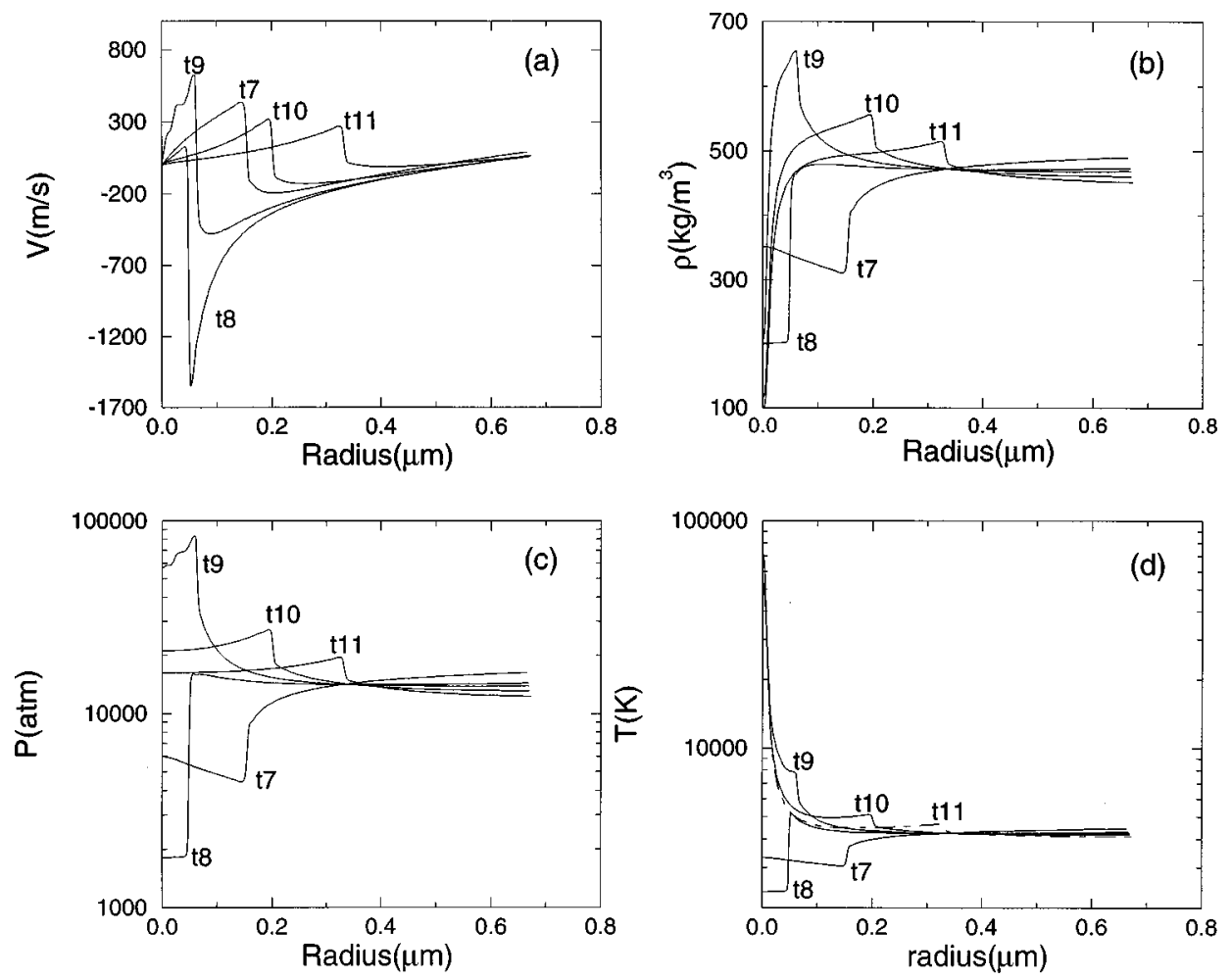

FIG. 3. Same as Fig. 2, but with time starting from $t_{7} ; t_{7}=19.265059 \mu \mathrm{s}, t_{8}=19.265104 \mu \mathrm{s}, t_{9}=19.265117 \mu \mathrm{s}, t_{10}$ $=19.265143 \mu \mathrm{s}, t_{11}=19.265174 \mu \mathrm{s}$.
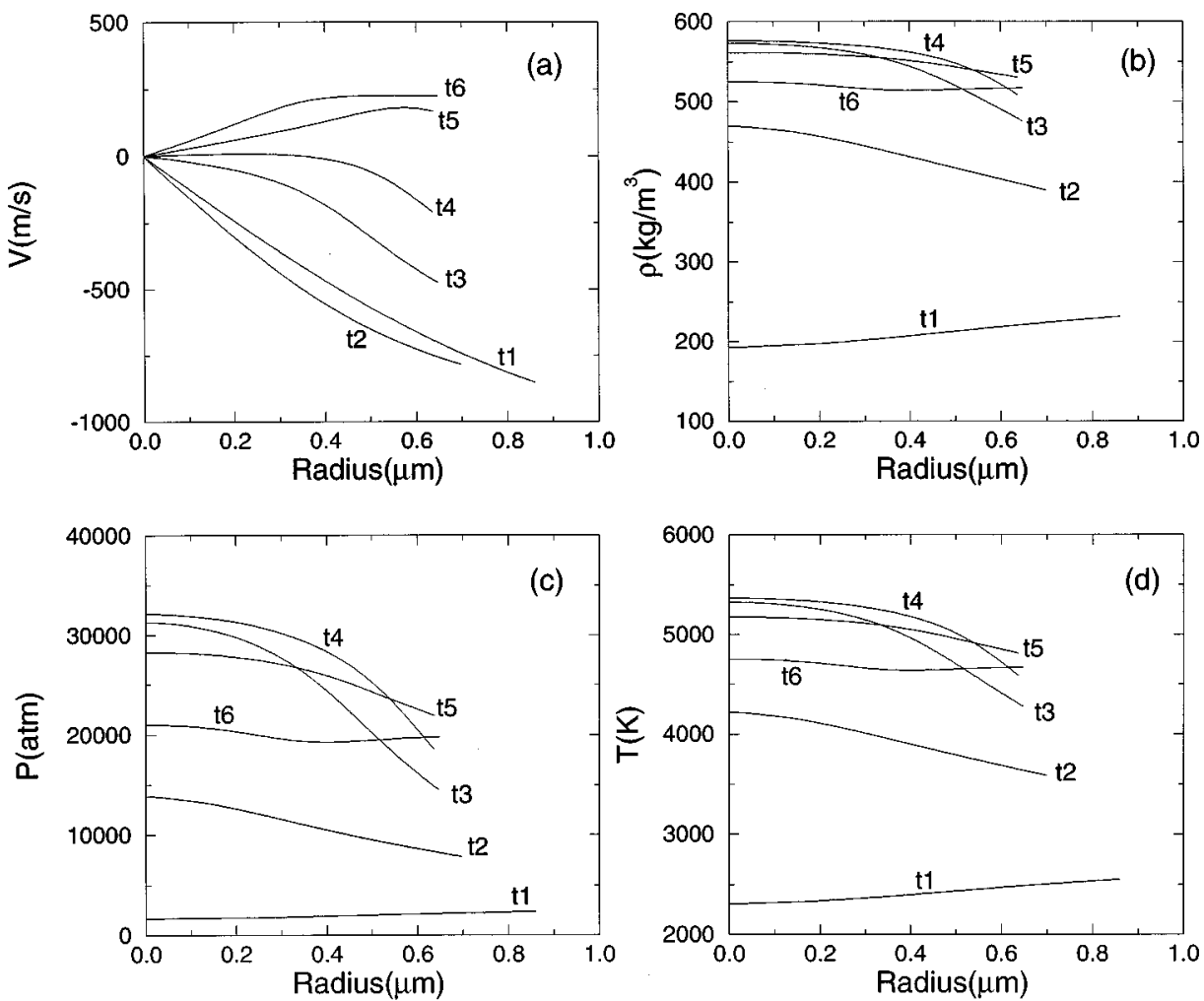

FIG. 4. Same as Fig. 2, but with Euler-RP2. Snapshots are shown at $t_{1}=19.284247 \mu \mathrm{s}, t_{2}=19.284438 \mu \mathrm{s}, t_{3}=19.284515 \mu \mathrm{s}, t_{4}$ $=19.284544 \mu \mathrm{s}, t_{5}=19.284590 \mu \mathrm{s}$, and $t_{6}=19.284643 \mu \mathrm{s}$. 

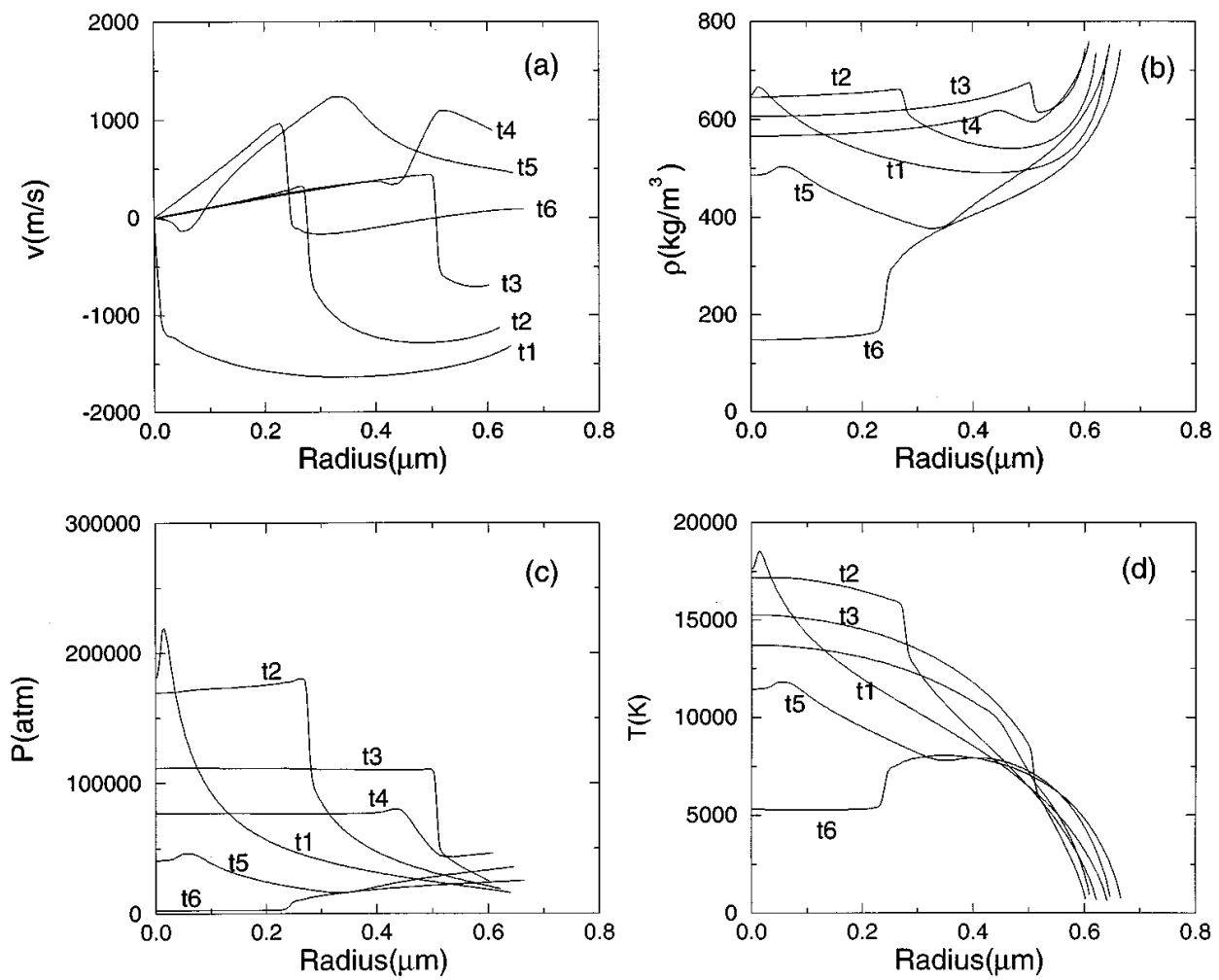

FIG. 5. Same as Fig. 2, but with NS-RP1. Snapshots are shown at $t_{1}=20.044169 \mu \mathrm{s}, t_{2}=20.044185 \mu \mathrm{s}, t_{3}=20.044206 \mu \mathrm{s}, t_{4}$ $=20.044227 \mu \mathrm{s}, t_{5}=20.044282 \mu \mathrm{s}$, and $t_{6}=20.044356 \mu \mathrm{s}$.

eral instants near $R_{\min }$ are shown for the NS-RP3 solution with VEOS. Although the compressional wave is very steep, it still cannot develop into a sharp shock.

The lack of shock at $P_{a}=1.35$ atm in the NS-RP3 with VEOS result is rather perplexing. We tested upwind and
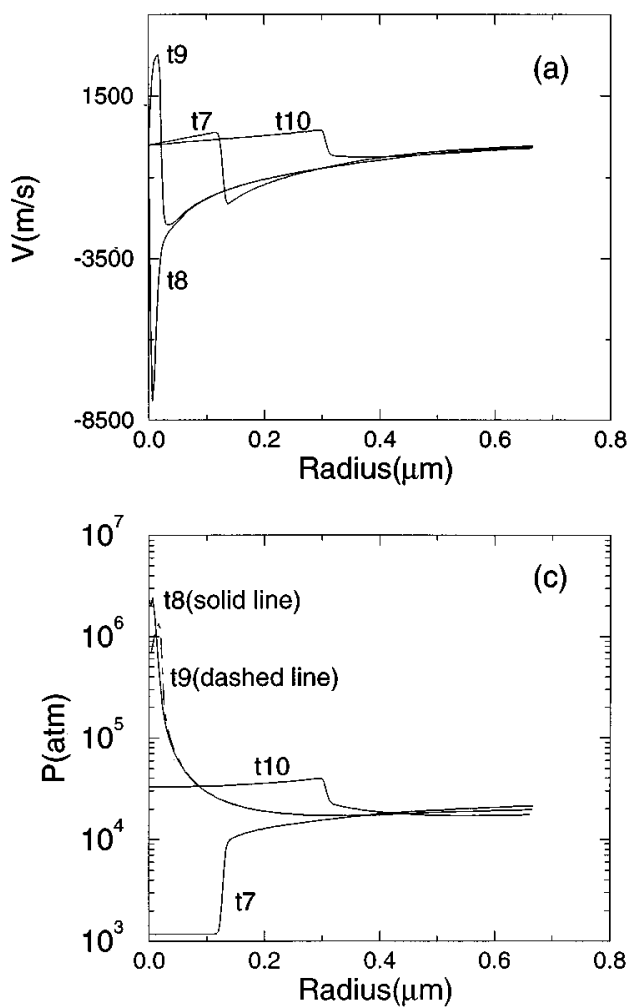

symmetric TVD schemes with all the limiter functions listed in Appendix A, the result is qualitatively the same. We also used Flynn's RP form [8], which includes a second-order compressibility correction to the Keller form analogous to the Gilmore form. The NS solution with Flynn's form is also
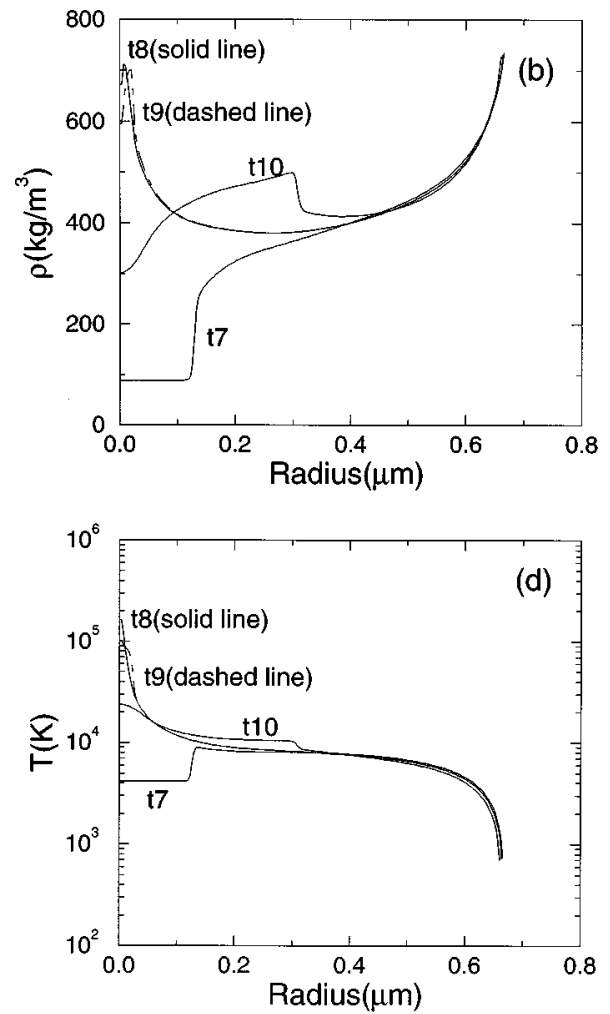

FIG. 6. Same as Fig. 5, but with time starting from $t_{7} ; t_{7}=20.044402 \mu \mathrm{s}, t_{8}=20.0444278 \mu \mathrm{s}, t_{9}=20.0444280 \mu \mathrm{s}, t_{10}$ $=20.044470 \mu \mathrm{s}$. 

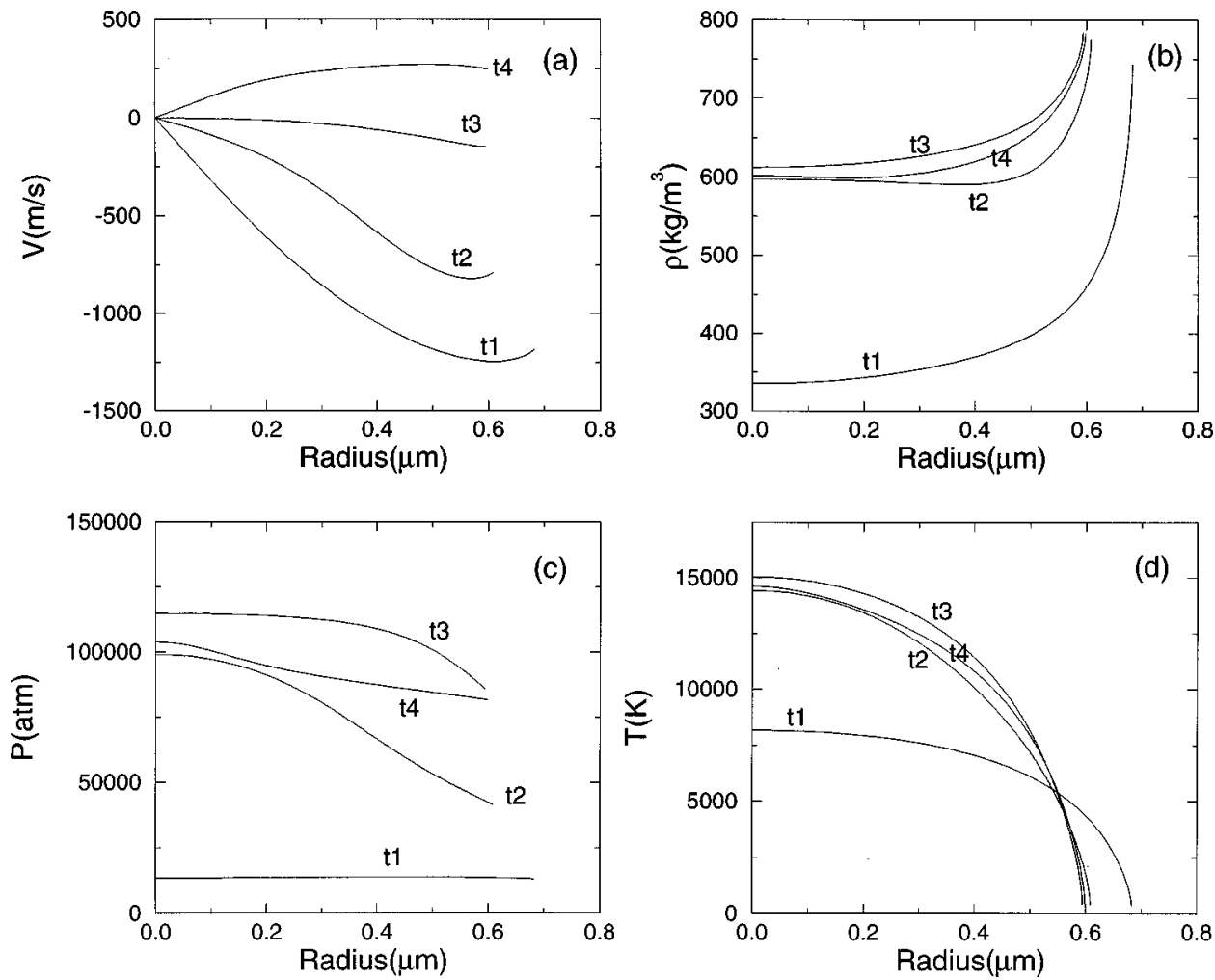

FIG. 7. Same as Fig. 2, but with NS-RP3. Snapshots are shown at $t_{1}=20.063662 \mu \mathrm{s}, t_{2}=20.063732 \mu \mathrm{s}, t_{3}=20.063760 \mu \mathrm{s}$, and $t_{4}$ $=20.063799 \mu \mathrm{s}$.

shock-free. This suggests that either additional liquid compressibility correction or the usage of RP3 does weaken the bubble's collapse, and sharp shocks cannot easily develop inside the bubble.

\section{B. Heat conduction}

We compare the Euler results with the Navier-Stokes results to reveal the effect of heat conduction in the gas and the liquid on the bubble's motion and the gas dynamics inside.

Figure 9 shows the $R(t)$ curves at $P_{a}=1.275 \mathrm{~atm}$ computed by the Euler and the NS equations. We can see that obvious differences exist between the Euler and the NS result, but not among the various forms of the RP equation. The computed $R(t)$ curves at $P_{a}=1.35$ atm are shown in Fig. 10. The NS result is closer to the experimental values [17] than the Euler result.
By turning off the viscosity in the NS solution, which becomes an inviscid solution with heat conduction, the $R(t)$ curves do not vary much. This reveals that the bubble-wall motion is affected mainly by heat conduction inside the bubble and across the bubble wall, and viscosity plays a much less important role. By monitoring the time history of the spatial profiles of the temperature inside, we find that the interface temperature is kept around the ambient temperature, and throughout most of its oscillation the bubble is nearly isothermal. Compared to an adiabatic expansion, a higher temperature inside the bubble is maintained during the expansion phase of each cycle, and hence a larger maximum radius $R_{\max }$ is reached.

As a consequence of the larger maximum radius, the gas in the bubble will gain more energy from the contraction of the displaced water in the later collapse stage. The larger expansion ratio $R_{\max } / R_{0}$ generally leads to an increase in the

TABLE II. Extrema values for air bubble collapse, $R_{0}=4.5 \mu \mathrm{m}, P_{a}=1.35 \mathrm{~atm}, f=26.4 \mathrm{kHz}$.

\begin{tabular}{lccccccc}
\hline \hline Gas dynamics & $\begin{array}{c}\mathrm{RP} \\
\text { form }\end{array}$ & $\begin{array}{c}T_{\max } \\
\left(10^{5} \mathrm{~K}\right)\end{array}$ & $\begin{array}{c}P_{\max } \\
\left(10^{6} \mathrm{~atm}\right)\end{array}$ & $\begin{array}{c}\dot{R}_{\max } \\
\left(\mathrm{km} \mathrm{s}^{-1}\right)\end{array}$ & $\begin{array}{c}R_{\min } \\
(\mu \mathrm{m})\end{array}$ & $\begin{array}{c}R_{\max } \\
(\mu \mathrm{m})\end{array}$ & Shock wave \\
\hline \multirow{3}{*}{ Euler (adiabatic) } & $\mathrm{RP1}$ & 3540 & 6641 & 2.10 & 0.589 & 38.93 & Yes \\
& $\mathrm{RP2}$ & 27.9 & 494.8 & 1.41 & 0.596 & 39.01 & Yes \\
& $\mathrm{RP3}$ & 3.50 & 372.0 & 1.53 & 0.583 & 39.00 & Yes \\
NS & $\mathrm{RP1}$ & 495 & 13780 & 2.50 & 0.581 & 43.79 & Yes \\
& $\mathrm{RP2}$ & 1.43 & 9.041 & 1.59 & 0.594 & 43.87 & Yes \\
NS (MEOS) & $\mathrm{RP3}$ & 0.249 & 0.464 & 1.74 & 0.579 & 43.87 & No \\
\hline \hline
\end{tabular}



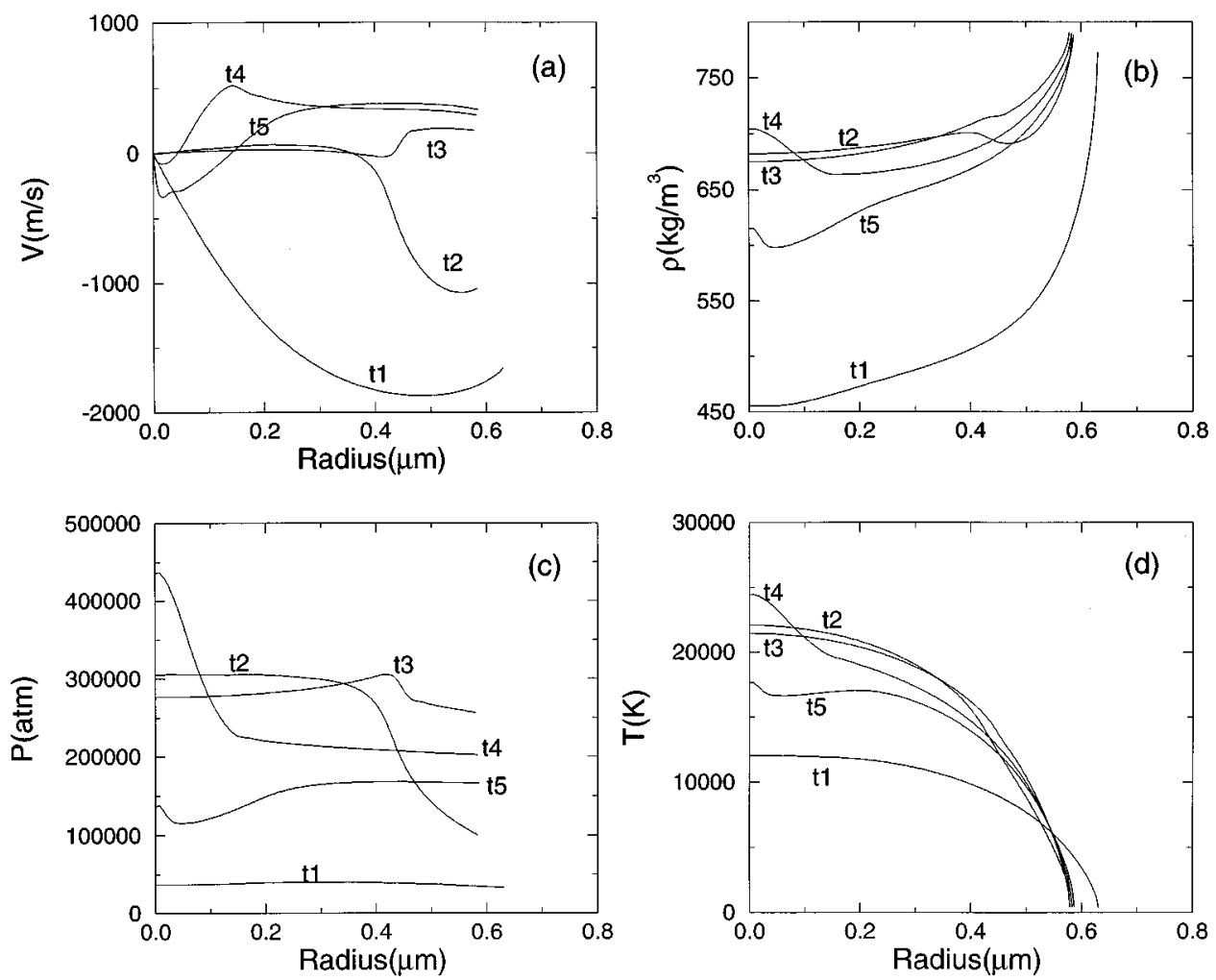

FIG. 8. Spatial profiles of (a) velocity, (b) density, (c) pressure, and (d) temperature, for an air bubble calculated with $R_{0}=4.5 \mu \mathrm{s}, f$ $=26.4 \mathrm{kHz}, \sigma=0.0725 \mathrm{~kg} \mathrm{~s}^{-2}$, and $P_{a}=1.35 \mathrm{~atm}$, with NS-RP3. Snapshots are shown at $t_{1}=21.305099 \mu \mathrm{s}, t_{2}=21.305131 \mu \mathrm{s}, t_{3}$ $=21.305142 \mu \mathrm{s}, t_{4}=21.305159 \mu \mathrm{s}$, and $t_{5}=21.305172 \mu \mathrm{s}$.

maximum temperature during the bubble's contraction, which is pointed out by Barber et al. [1] and Löfstedt et al. [17].

Recalling Tables I and II, we see that the bubble's maximum radius or interface velocity in NS results is greater than Euler results, so are the thermal peaks when no shock waves are formed. However, when sharp shock wave develops as in $P_{a}=1.35 \mathrm{~atm}$, thermal peaks of the Euler results are greater. At this time, both viscosity and heat conduction inside the gas smear the discontinuity and thus reduce the thermal

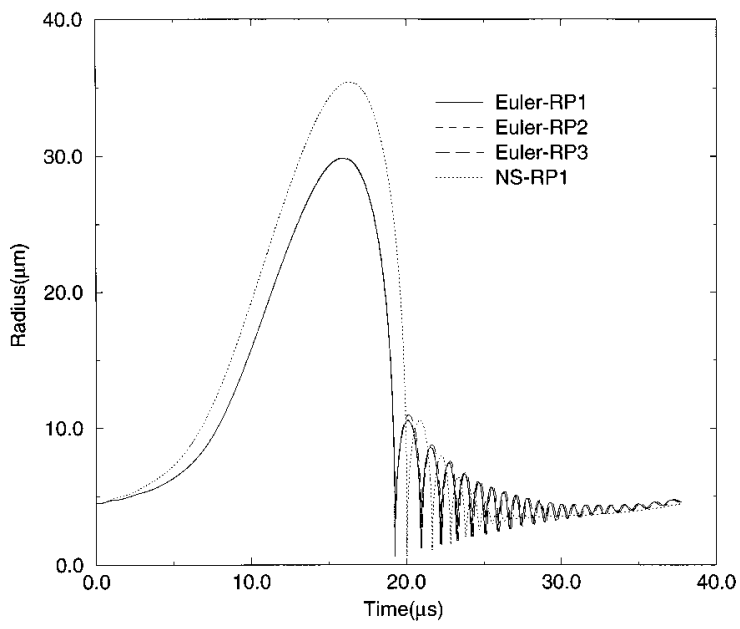

FIG. 9. Comparison of the bubble radius vs time in one period for several hydrodynamics models, with $R_{0}=4.5 \mu \mathrm{m}, P_{a}$ $=1.275 \mathrm{~atm}, f=26.4 \mathrm{kHz}, \sigma=0.0725 \mathrm{~kg} \mathrm{~s}^{-2}$, for an air bubble. peaks. This will be further demonstrated in the argon bubble case, which was studied by Vuong and Szeri [15], at $P_{a}$ $=1.3 \mathrm{~atm}$. We recalculated it using both Euler-RP1 and NSRP1 models. In order to compare with Vuong and Szeri's results, we also set the surface tension to zero. The Euler solution in Fig. 11 shows the time evolution of gas variable distributions. We see that the collapsing bubble launches a shock wave toward the center at $t_{2}$. On the other hand, the NS solution in Fig. 12 shows that only compressional waves

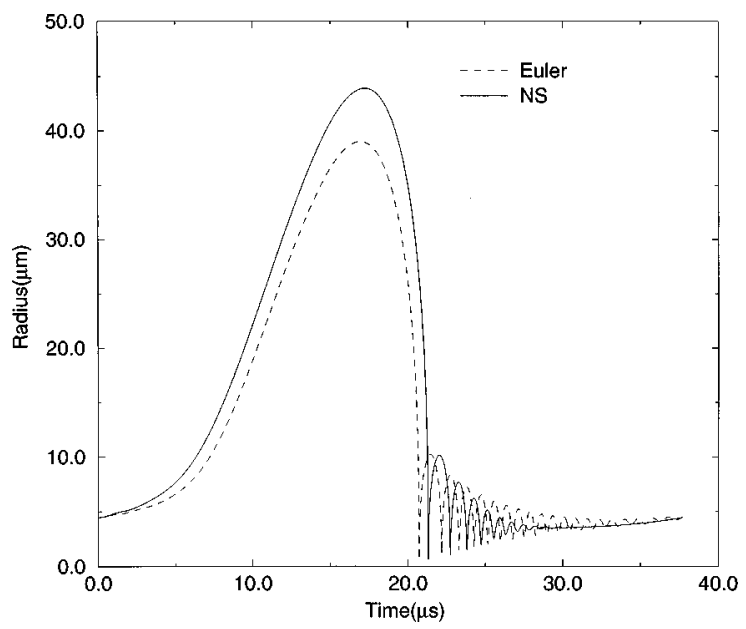

FIG. 10. Comparison of the bubble radius vs time in one period for NS-RP3 (solid line) and Euler-RP3 (dashed line), with $R_{0}$ $=4.5 \mu \mathrm{m}, P_{a}=1.275 \mathrm{~atm}, f=26.4 \mathrm{kHz}, \sigma=0.0725 \mathrm{~kg} \mathrm{~s}^{-2}$, for an air bubble. 

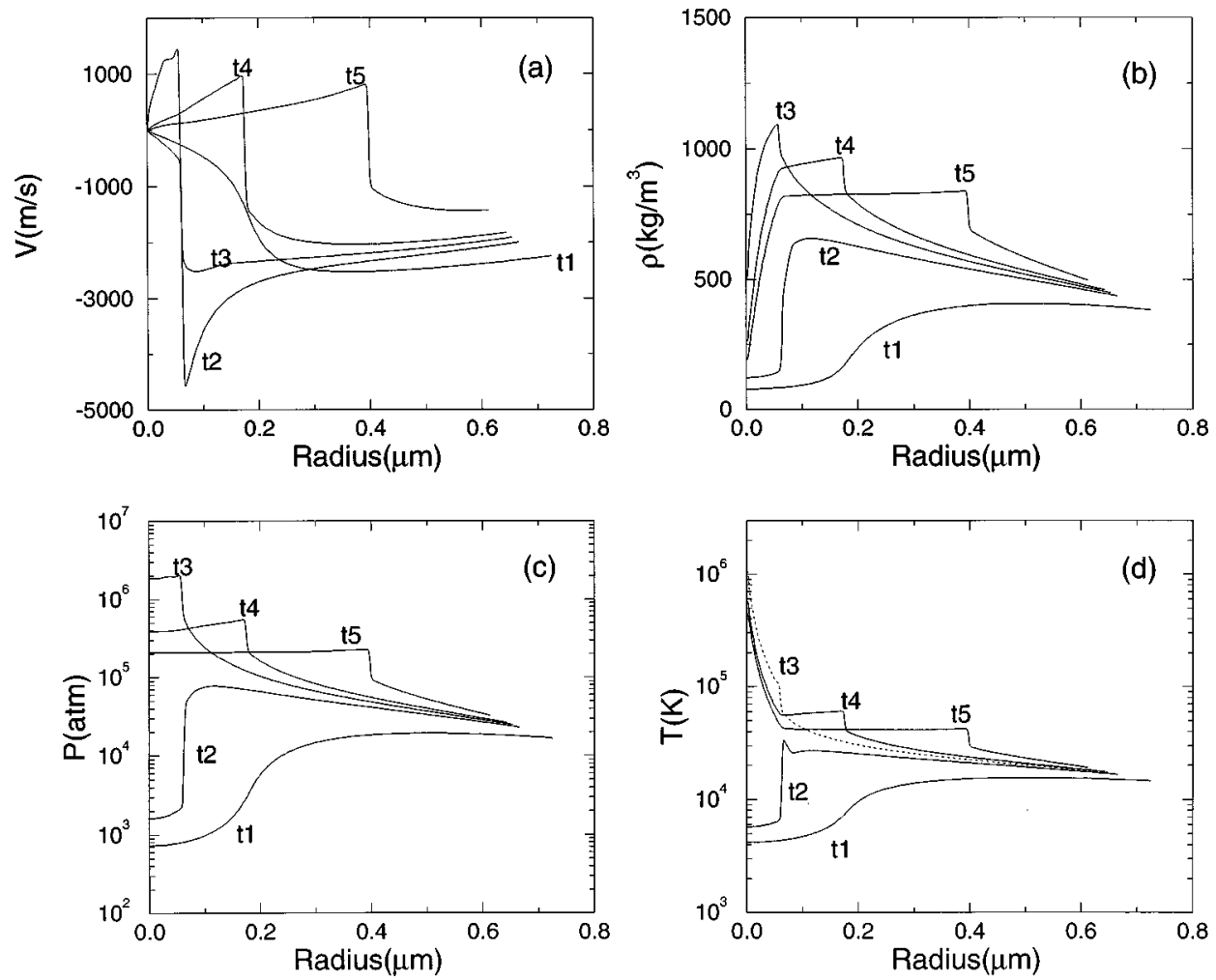

FIG. 11. Spatial profiles of (a) velocity, (b) density, (c) pressure, and (d) temperature, for an argon bubble calculated with $R_{0}$ $=4.5 \mu \mathrm{m}, f=26.4 \mathrm{kHz}, \sigma=0$, and $P_{a}=1.3 \mathrm{~atm}$, with Euler-RP1. Snapshots are shown at $t_{1}=21.364915 \mu \mathrm{s}, t_{2}=21.364943 \mu \mathrm{s}, t_{3}$ $=21.364949 \mu \mathrm{s}, t_{4}=21.364954 \mu \mathrm{s}$, and $t_{5}=21.364973 \mu \mathrm{s}$.
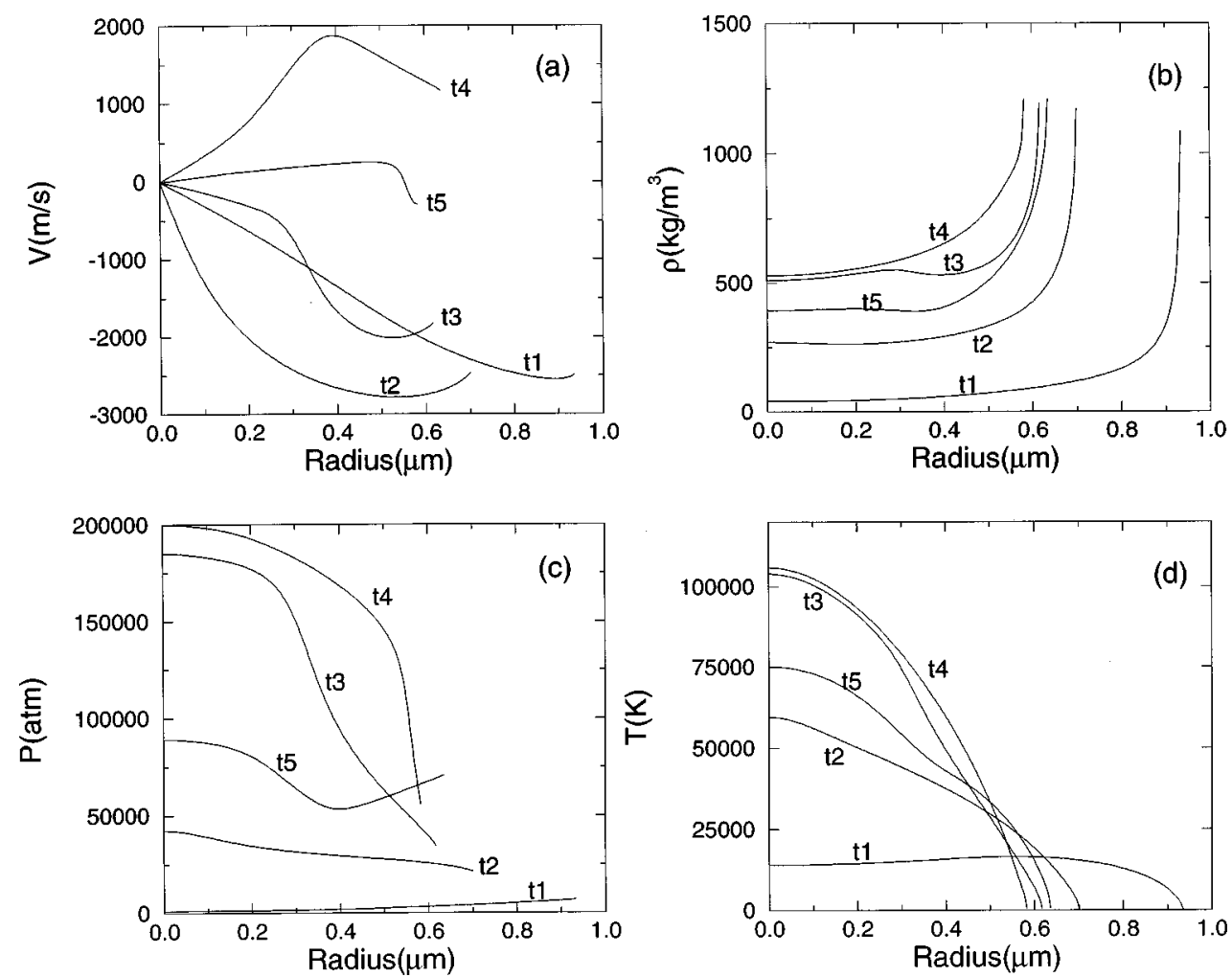

FIG. 12. Same as Fig. 11, but with NS-RP1. $t_{1}=21.746346 \mu \mathrm{s}, t_{2}=21.746437 \mu \mathrm{s}, t_{3}=21.746475 \mu \mathrm{s}, t_{4}=21.746503 \mu \mathrm{s}$, and $t_{5}$ $=21.746551 \mu \mathrm{s}$. 
are produced (for example, see the velocity profiles), but no shock wave is formed.

\section{EOS}

We have used both VEOS and MEOS in an air bubble to study the effect of EOS. The main difference between them is that VEOS employs an excluded volume that forbids the gas density from reaching a maximum value (about $794 \mathrm{~g} \mathrm{~cm}^{-3}$ for air), while MEOS includes vibrational excitation, dissociation, ionization, and a repulsive and attractive intermolecular potential.

A dense, cold layer near the bubble wall is formed when heat conduction is included and the driving pressure is high enough, as pointed out by Chu and Leung [10]. This can also be seen from the spatial distributions of density and temperature in Figs. 5-8. This layer is also formed when using MEOS. We find that when the dense cold layer is formed, the negative (attractive) intermolecular potential exceeds the first term in pressure formula in MEOS, making pressure negative near the interface. This indicates that the MEOS is not applicable at low temperature and high density. This situation does not occur in VEOS.

To avoid the negative pressure in MEOS discussed above, we used a coarser grid in the water zone that does not resolve the thermal boundary layer in water. This artificially reduced the heat transfer between air and water and smeared the sharp gradients of the dense cold layer. Although this may result in a higher temperature near the interface, it does not change the gas dynamics much elsewhere, especially the shock formation near the bubble center. Furthermore, we used the same coarse grid in VEOS for comparison purpose.

For comparing the effects of VEOS and MEOS, we can compare the results listed in the last two rows of Table I and II, respectively. We see that MEOS gives much higher peak temperature and extremely high pressure, behaving much like ideal gas EOS. The minimum radius is smaller than that of VEOS and closer to that obtained with an ideal gas EOS without excluded volume. As a result of this comparison, we conclude that it is easier to obtain shock waves with MEOS than with VEOS. It is not clear though which EOS is more realistic.

\section{Surface tension}

Many authors have stressed the importance of the surface tension in bubble dynamics [1,6,7]. The surface tension acts as an inward pressure on the bubble. Therefore, a decrease in surface tension will give rise to a higher expansion ratio $R_{\max } / R_{0}$, as shown in [1] and also our calculations. The gas dynamics during the bubble's collapse stage is therefore considerably affected by the surface tension. However, how surface tension affects the gas dynamics, especially the shock formation, has not been discussed before to our knowledge. In some previous calculations of the gas dynamics $[3,4,15]$, the surface tension term was ignored, and this certainly would overestimate the collapse strength.

We found that the shock wave formation is very sensitive to the value of surface tension. To demonstrate this, we recomputed the air bubble at $P_{a}=1.35$ atm using NS-RP3 with a slightly smaller surface tension of $\sigma=0.05 \mathrm{~kg} \mathrm{~s}^{-2}$. This value was typically used in [1] to account for the impurities in water. We also find that taking the value of surface tension $\sigma=0.05 \mathrm{~kg} \mathrm{~s}^{-2}$, instead of the standard value $0.0725 \mathrm{~kg} \mathrm{~s}^{-2}$, will make the $R-t$ curve fit the experimental data better. With $\sigma=0.05 \mathrm{~kg} \mathrm{~s}^{-2}$, a shock wave develops. The peak temperature reaches as high as $345000 \mathrm{~K}$.

We also recomputed the case of the argon bubble at $P_{a}$ $=1.3 \mathrm{~atm}$, which Vuong and Szeri [15] studied previously. Firstly, for comparing with Fig. 11, we again used Euler-RP1 model, but with standard surface tension. Figure 13 shows the Euler result with nonzero surface tension, where no shock is formed. Secondly, we used the NS-RP1 model just as Vuong and Szeri did but with a standard surface tension $0.0725 \mathrm{~kg} \mathrm{~s}^{-2}$. The center peak temperature is considerably reduced to $51980 \mathrm{~K}$ from $108230 \mathrm{~K}$ with zero surface tension.

Our study shows that with other parameters fixed, shock waves can be formed easily if the surface tension is reduced. The inclusion of surface tension also weakens the strength of the collapse in the shock-free case, as reflected by the peak values of temperature, pressure, radiated power, etc. This further emphasizes the importance of surface tension both in bubble cavitation and in SL.

\section{E. Bubble contents}

Recently there has been a conjecture that sonoluminescing air bubbles rectify argon [28]. This mechanism explains the existence of an experimentally stable SL bubble that is diffusively unstable according to mass diffusion theory. The numerical study of Vuong and Szeri [15] has found that in pure noble gas bubble, no shock waves but wavy disturbances develop during the final stage of the collapse for $P_{a}$ ranging from 1.2 to $1.3 \mathrm{~atm}$. Motivated by these studies, we made comparison between air bubble and argon bubble.

We note that the ratio of specific heats is different between air and argon. The larger ratio of specific heats of argon makes its temperature higher upon compression, and shock waves do not develop easily in noble gases. For example, for argon bubble (Fig. 13) at $P_{a}=1.3 \mathrm{~atm}$, no shock is formed, while for an air bubble at a lower $P_{a}$ $=1.275$ atm (Fig. 2 and Fig. 3), a shock has already developed. With diffusive transport included and the adoption of a more accurate RP equation (RP3), no shock wave is formed in the air bubble up to $P_{a}=1.35 \mathrm{~atm}$ with standard surface tension and VEOS. For a noble gas bubble, when VEOS is used, it seems even more unlikely that shock waves will be formed because the diffusive transport coefficients become larger at higher temperature.

However, whether there are sharp shocks or not does not change the result that at larger $P_{a}$ the temperature inside the bubble can be high enough to emit light. We proceed to discuss the shock-unrelated model for SL in the following section.

\section{F. Existence of shock-free picosecond pulse}

One popular idea to explain the highly energetic and short light pulse in SL is that a focusing shock wave developed in the bubble during the collapse stage [2-5]. However, previous simulations that supported this idea have made various 

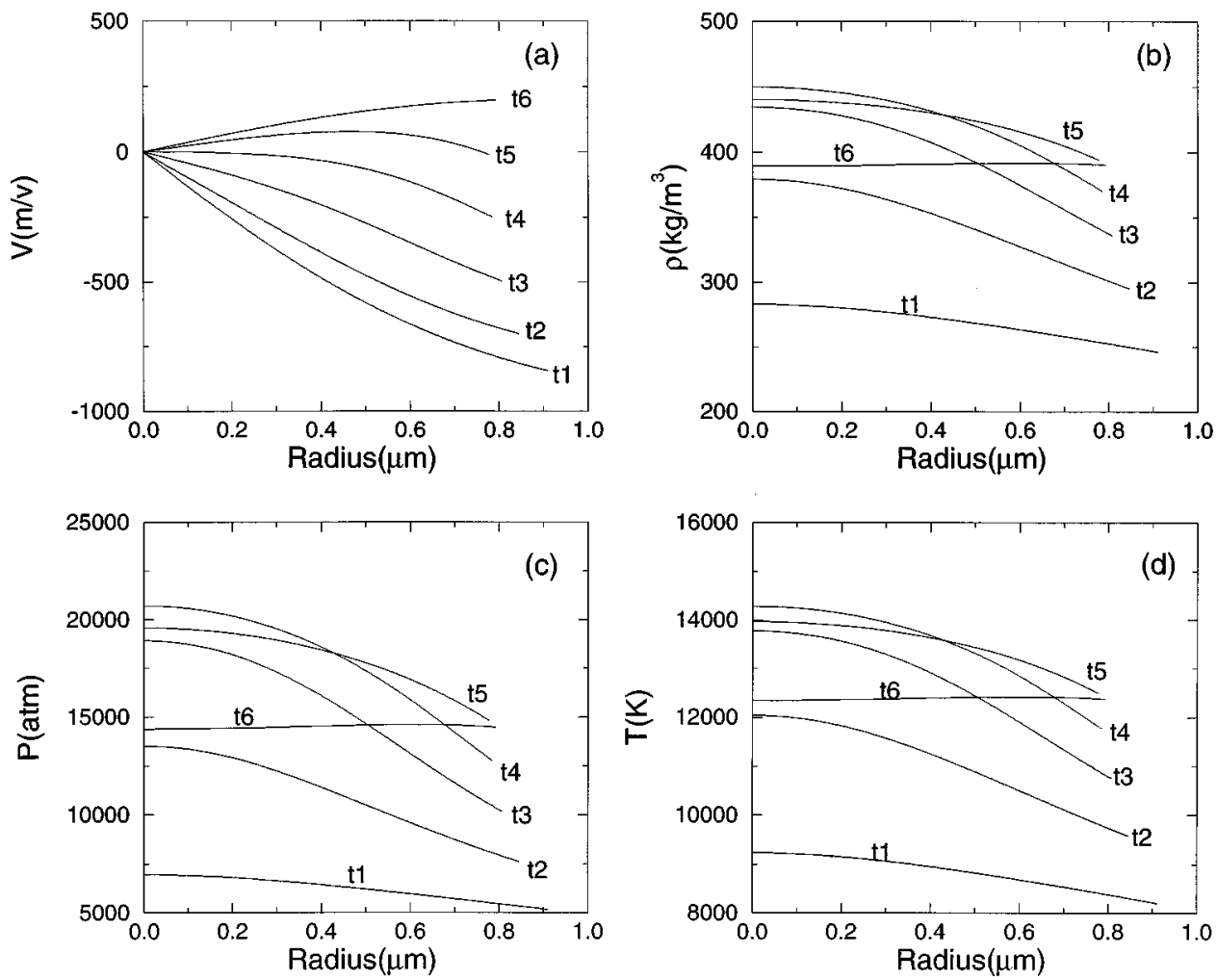

FIG. 13. Same as Fig. 11, but with $\sigma=0.0725 \mathrm{~kg} \mathrm{~s}^{-2}$. Snapshots are shown at $t_{1}=19.478161 \mu \mathrm{s}, t_{2}=19.478243 \mu \mathrm{s}, t_{3}$ $=19.478308 \mu \mathrm{s}, t_{4}=19.478365 \mu \mathrm{s}, t_{5}=19.478421 \mu \mathrm{s}$, and $t_{6}=19.478542 \mu \mathrm{s}$.

assumptions. Our present simulation reproduces all these shock wave results when we make these same assumptions. Furthermore, our calculation of air and argon bubble (as well as that of Vuong and Szeri [15]), using other hydrodynamic models including a more realistic NS-RP3 model, reveals the sensitive dependence of shock formation on the liquid compressibility, diffusive transport, surface tension, as well as the EOS. Our results call into question whether the development of shock waves is really necessary for sonoluminescence.

For demonstration purpose, we use a blackbody radiation model for the emitted power [Eq. (20)]. Figure 14 shows the time history of the temperature at $r=0\left(T_{c}\right)$ and the total radiation power $\dot{E}(t)$ for air bubble at $P_{a}=1.275 \mathrm{~atm}$. The condition $T_{c} \approx 12000 \mathrm{~K}$ lasts about $120 \mathrm{ps}$, which is in agreement with previous numerical results $[4,15]$. However, because the peak temperature at a point does not contribute much to the integrated power, the power pulse is narrower than that of the peak temperature as shown. The peak power is about $0.58 \mathrm{~mW}$ with a FWHM of about 100 ps.

In Fig. 15 the time history of $T_{c}$ and the average power are given for the $P_{a}=1.35 \mathrm{~atm}$ air bubble. We again see that temperature above $12000 \mathrm{~K}$ can last more than $200 \mathrm{ps}$, while the power pulse is much narrower. Its peak is about $2.3 \mathrm{~mW}$ with a FWHM of about $50 \mathrm{ps}$. The wiggle in the temperature is related to the reflection of compressional waves at the center. In Fig. 16 the result of MEOS is shown. In this case a sharp peak is developed, and the power can reach $4.8 \mathrm{~mW}$ with its FWHM lasting only about 5 ps.

For an argon bubble, we first show the result by using RP1 without surface tension in Fig. 17 and a more realistic result in Fig. 18. We can see from Fig. 17 that the $T_{c}$ is quite similar to that obtained by Vuong and Szeri [15]. The peak temperature is $108230 \mathrm{~K}$, close to their $118240 \mathrm{~K}$. The difference is quite small in view of the different numerical methods used. The peak power is about $600 \mathrm{~mW}$, much larger than the experimental value, and its FWHM is about 60 ps. For the NS-RP3 result shown in Fig. 18, the peak radiation power is about $68 \mathrm{~mW}$ with a FWHM of nearly 200 ps.

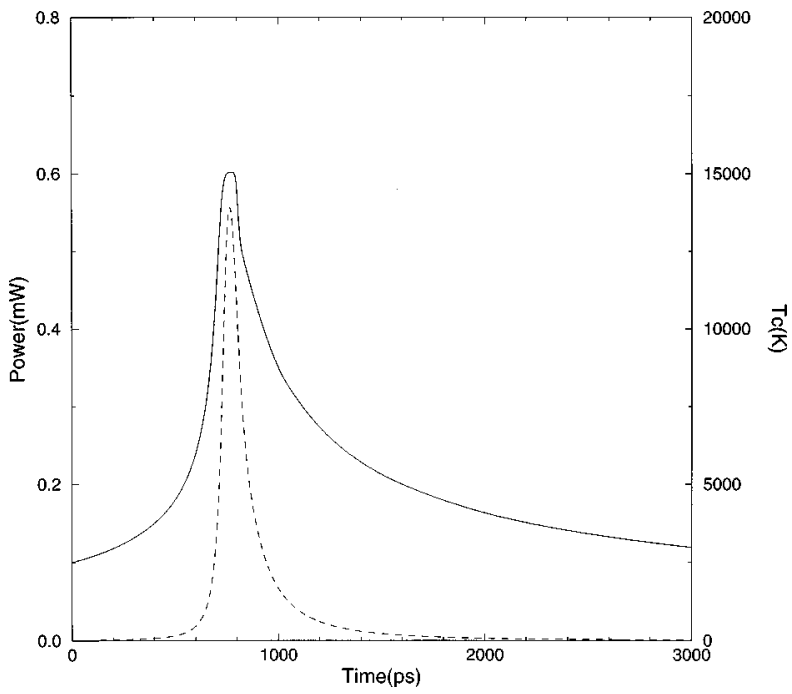

FIG. 14. Temperature at the bubble center (solid line) and radiation power (dashed line) vs time, for an air bubble with $R_{0}$ $=4.5 \mu \mathrm{m}, P_{a}=1.275 \mathrm{~atm}, f=26.4 \mathrm{kHz}, \sigma=0.0725 \mathrm{~kg} \mathrm{~s}^{-2}$, using NS-RP3-VEOS. The starting time is $t_{0}=20.063 \mu \mathrm{s}$. 


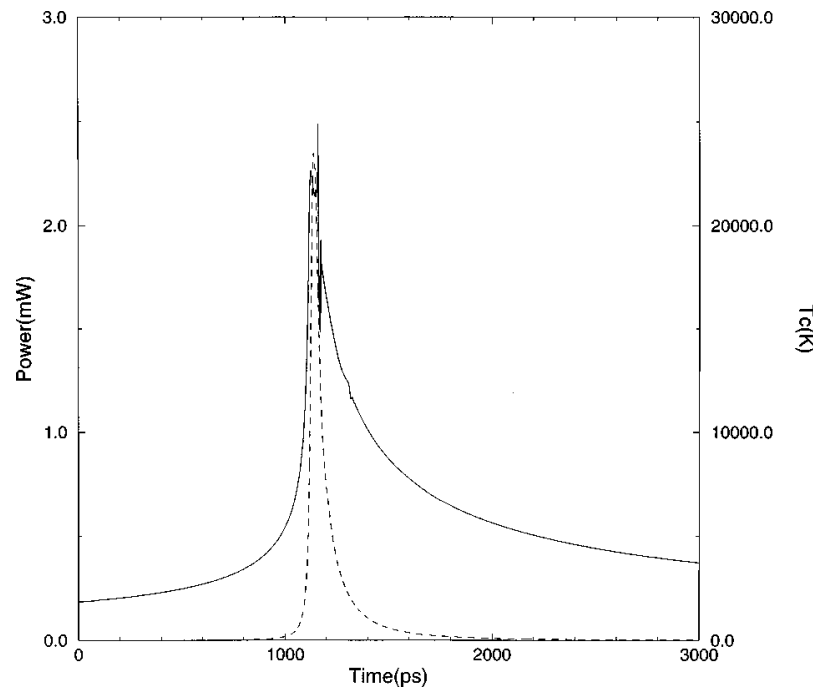

FIG. 15. Same as Fig. 14, but with $P_{a}=1.35$ atm. The starting time is $t_{0}=21.304 \mu \mathrm{s}$.

The above results show that even without the focusing of shock waves, the total power radiated by the bubble content can be on the order of 1-100 mW with a FWHM of about $100 \mathrm{ps}$, which is already comparable to the experimental result. On the other hand, the power pulse caused by shocks is much shorter, of less than a few ps. This indicates that in hydrodynamic theory, shock wave model alone is not adequate for the explanation of SL. In fact, an alternative mechanism for producing high temperature leading to sonoluminescence by focusing of wavy disturbances, or continuous compressional waves in our terminology seems to be more robust than shock wave focusing model. The role of these continuous compressional waves is even more important if the hypothesis that "SL air bubble rectifies argon", [28] is proved to be true.

\section{CONCLUSIONS}

We have shown the important effects of the liquid compressibility, heat conduction, surface tension, the bubble con-

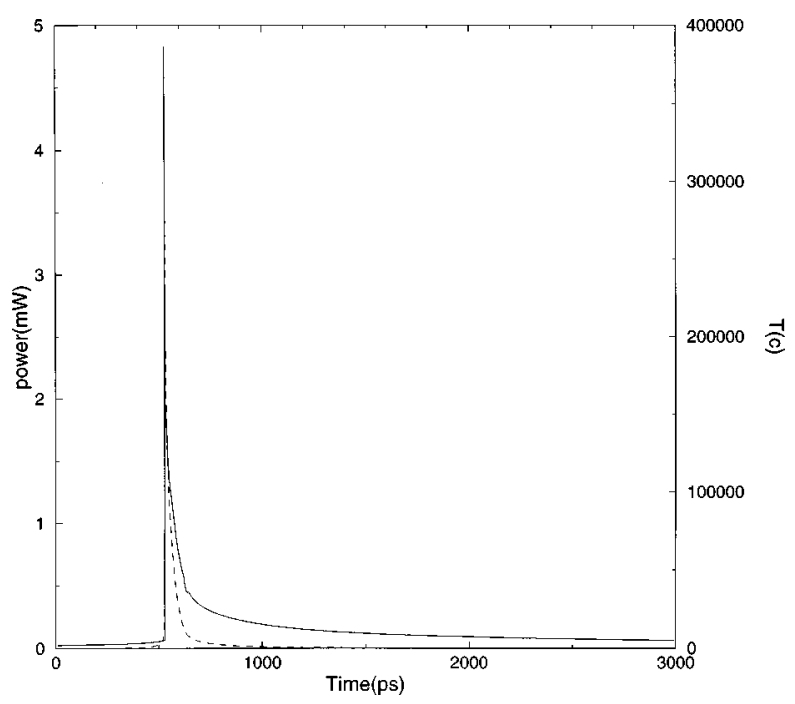

FIG. 16. Same as Fig. 15, but with NS-RP3-MEOS. The starting time is $t_{0}=21.304 \mu \mathrm{s}$.

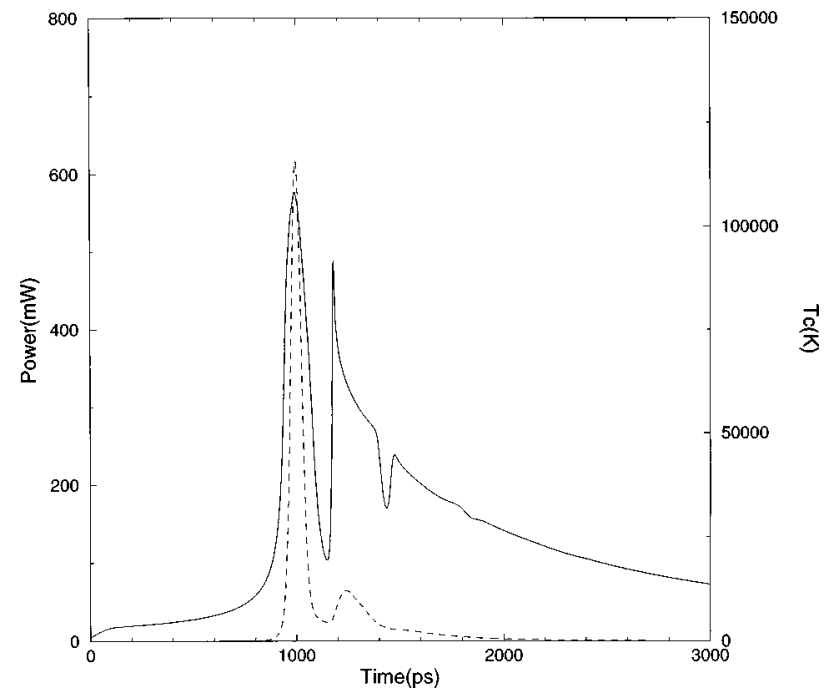

FIG. 17. Temperature at the bubble center (solid line) and radiation power (dashed line) vs time, for an argon bubble with $R_{0}$ $=4.5 \mu \mathrm{m}, \quad P_{a}=1.3 \mathrm{~atm}, f=26.4 \mathrm{kHz}, \sigma=0$, using NS-RP1VEOS. The starting time is $t_{0}=21.7455 \mu \mathrm{s}$.

tent, and gas EOS on the gas dynamics of a sonoluminescing bubble by comparing the numerical results of the Euler and Navier-Stokes equations coupled to various forms of the RP equation. Even though the effect of liquid compressibility is important only during a very short duration of bubble collapse, it can damp out shock waves and make the solution shock-free. Surface tension has the same effect as liquid compressibility to reduce the violence of the bubble motion. On the other hand, heat conduction between liquid and gas is important during the slow expansion phase, as it leads to a larger expansion ratio $R_{\max } / R_{0}$, enhancing the violence of the bubble collapse. Our Navier-Stokes solutions, taking into account heat conduction, surface tension, and liquid compressibility indicate that even without shock waves, the reflection of the continuous compressional waves from the bubble center is enough to focus the energy so that a short

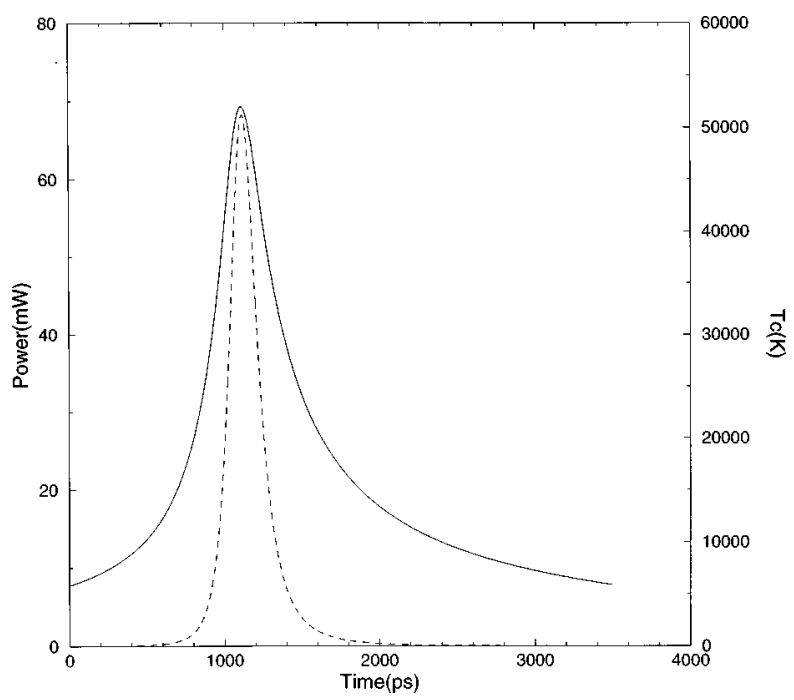

FIG. 18. Same as Fig. 17, but with $\sigma=0.0725 \mathrm{~kg} \mathrm{~s}^{-2}$ and NSRP3-VEOS. The starting time is $t_{0}=20.3155 \mu \mathrm{s}$. 
duration of high temperature condition within the bubble is reached, with quantities comparable to experimental values. A new hydrodynamic model taking into account the low temperature, high density EOS for the gas, and a more accurate representation of the liquid compressibility may better predict the bubble motion and various thermodynamic properties at conditions corresponding to sonoluminescence.

\section{ACKNOWLEDGMENTS}

We acknowledge the support of a Hong Kong Research Grants Council Grant No. CUHK 312/96P and a Chinese University Direct Grant (Project ID: 2060093). L.Y. thanks the Chinese University for financial support.

\section{APPENDIX A: NUMERICAL METHOD FOR THE GAS EQUATIONS IN THE BUBBLE}

The predictor-corrector method applied to the hyperbolic conservation laws Eq. (24) is as follows. The predictor step is

$$
\overline{\mathbf{Q}}=\mathbf{Q}^{n}-\lambda\left(\hat{\mathbf{F}}_{i+1 / 2}^{n}-\hat{\mathbf{F}}_{i-1 / 2}^{n}\right)+\Delta t \mathbf{S}\left(\mathbf{Q}^{n}\right)+\Delta t \mathbf{S}_{v}\left(\mathbf{Q}^{n}\right)
$$

whereas the corrector is

$$
\begin{aligned}
\mathbf{Q}^{n+1}= & \mathbf{Q}^{n}-0.5\left[\lambda\left(\hat{\mathbf{F}}_{i+1 / 2}^{n}-\hat{\mathbf{F}}_{i-1 / 2}^{n}\right)-\Delta t \mathbf{S}\left(\mathbf{Q}^{n}\right)-\Delta t \mathbf{S}_{v}\left(\mathbf{Q}^{n}\right)\right. \\
& \left.+\lambda\left(\overline{\mathbf{F}}_{i+1 / 2}-\overline{\mathbf{F}}_{i-1 / 2}\right)-\Delta t \mathbf{S}(\overline{\mathbf{Q}})-\Delta t \mathbf{S}_{v}(\overline{\mathbf{Q}})\right],
\end{aligned}
$$

where $\lambda=\Delta t / \Delta x$. The numerical flux $\hat{\mathbf{F}}_{i+1 / 2}$ is defined for the non-MUSCL formulation as [25]

$$
\hat{\mathbf{F}}_{i+1 / 2}=\frac{1}{2}\left[\mathbf{F}_{i}+\mathbf{F}_{i+1}+\mathbf{R}_{i+1 / 2} \boldsymbol{\Phi}_{i+1 / 2}\right] .
$$

Here $\mathbf{R}_{i+1 / 2}$ is a matrix whose column vectors are the right eigenvectors of the flux Jacobian $\partial \mathbf{F} / \partial \mathbf{Q}$ evaluated at some symmetric average of $\mathbf{Q}_{i}$ and $\mathbf{Q}_{i+1}$. We take a generalized Roe average of Vonokur [29] due to different EOSs encountered. Both Harten-Yee second-order upwind TVD scheme and Yee-Roe-Davis second-order symmetric TVD scheme are used. The $l$ th element of $\boldsymbol{\Phi}_{i+1 / 2}$ denoted by $\left(\phi_{i+1 / 2}^{l}\right)^{U}$ for Harten-Yee upwind scheme is

$$
\begin{aligned}
\left(\phi_{i+1 / 2}^{l}\right)^{U}= & \sigma\left(a_{i+1 / 2}^{l}\right)\left(g_{i}^{l}+g_{i+1}^{l}\right) \\
& -\psi\left(a_{i+1 / 2}^{l}+\gamma_{i+1 / 2}^{l}\right) \alpha_{i+1 / 2}^{l} .
\end{aligned}
$$

Here $a_{i+1 / 2}^{l}$ is the eigenvalue of the flux Jacobian $\partial \mathbf{F} / \partial \mathbf{Q}$ evaluated at some symmetric average of $\mathbf{Q}_{i}$ and $\mathbf{Q}_{i+1}, \alpha_{i+1 / 2}^{l}$ are elements of $\alpha_{i+1 / 2}=\mathbf{R}_{i+1 / 2}^{-1}\left(\mathbf{Q}_{i+1}-\mathbf{Q}_{i}\right)$, and $g_{i+1 / 2}^{l}$ are the limiter functions. The functions $\sigma(z)$ and $\gamma_{i+1 / 2}$ are defined as

$$
\begin{gathered}
\sigma(z)=\frac{1}{2}\left[\psi(z)-\lambda z^{2}\right] \\
\gamma_{i+1 / 2}^{l}=\sigma\left(a_{i+1 / 2}^{l}\right)\left\{\begin{array}{l}
\left(g_{i+1}^{l}-g_{i}^{l}\right) / \alpha_{i+1 / 2}^{l}, \quad \alpha_{i+1 / 2}^{l} \neq 0 \\
0, \quad \alpha_{i+1 / 2}^{l}=0 .
\end{array}\right.
\end{gathered}
$$

The function $\psi(z)$ is an entropy correction to $|z|$ defined as

$$
\psi(z)=\left\{\begin{array}{l}
|z|, \quad|z| \geqslant \delta_{1} \\
\left(z^{2}+\delta_{1}^{2}\right) / 2 \delta_{1}, \quad|z|<\delta_{1}
\end{array} .\right.
$$

Since the present problem contains only unsteady shocks that generally do not violate the entropy condition, $\delta_{1}$ is set to zero. Some applicable limiter functions $g_{i}^{l}$ are

$$
\begin{gathered}
g_{i}^{l}=\operatorname{minmod}\left(\alpha_{i-1 / 2}^{l}, \alpha_{i+1 / 2}^{l}\right), \\
g_{i}^{l}=\left(\alpha_{i-1 / 2}^{l} \alpha_{i+1 / 2}^{l}+\left|\alpha_{i-1 / 2}^{l} \alpha_{i+1 / 2}^{l}\right|\right) /\left(\alpha_{i-1 / 2}^{l}+\alpha_{i+1 / 2}^{l}\right), \\
g_{i}^{l}=\operatorname{minmod}\left[2 \alpha_{i-1 / 2}^{l}, 2 \alpha_{i+1 / 2}^{l}, 0.5\left(\alpha_{i-1 / 2}^{l}+\alpha_{i+1 / 2}^{l}\right)\right], \\
g_{i}^{l}=S \max \left[0, \min \left(2\left|\alpha_{i+1 / 2}^{l}\right|, S \alpha_{i-1 / 2}^{l}\right),\right. \\
\left.\min \left(\left|\alpha_{i+1 / 2}^{l}\right|, 2 S \alpha_{i-1 / 2}^{l}\right)\right], S=\operatorname{sgn}\left(\alpha_{i+1 / 2}^{l}\right) .
\end{gathered}
$$

The "minmod" function of a list of arguments gives a value equal to the smallest number in absolute value if the list of arguments is of the same sign, or equal to zero if any arguments are of opposite sign. For $\alpha_{i+1 / 2}+\alpha_{i-1 / 2}=0, g_{i}^{l}$ is set to zero in Eq. (A12).

The $l$ th element of $\boldsymbol{\Phi}_{i+1 / 2}$ denoted by $\left(\phi_{i+1 / 2}^{l}\right)^{S}$ for the Yee-Roe-Davis symmetric scheme is

$$
\begin{aligned}
\left(\phi_{i+1 / 2}^{l}\right)^{S}= & -\lambda\left(a_{i+1 / 2}^{l}\right)^{2} s_{i+1 / 2}^{l} \\
& -\psi\left(a_{i+1 / 2}^{l}\right)\left(\alpha_{i+1 / 2}^{l}-s_{i+1 / 2}^{l}\right) .
\end{aligned}
$$

Several limiter functions $s_{i+1 / 2}^{l}$ can be used such as

$$
\begin{gathered}
s_{i+1 / 2}^{l}=\operatorname{minmod}\left(\alpha_{i-1 / 2}^{l}, \alpha_{i+1 / 2}^{l}\right)+\operatorname{minmod}\left(\alpha_{i+1 / 2}^{l}, \alpha_{i+3 / 2}^{l}\right) \\
-\alpha_{i+1 / 2}^{l}, \\
s_{i+1 / 2}^{l}=\operatorname{minmod}\left(\alpha_{i-1 / 2}^{l}, \alpha_{i+1 / 2}^{l}, \alpha_{i+3 / 2}^{l}\right), \\
s_{i+1 / 2}^{l}=\operatorname{minmod}\left[2 \alpha_{i-1 / 2}^{l}, 2 \alpha_{i+1 / 2}^{l}, 2 \alpha_{i+3 / 2}^{l}, 0.5\left(\alpha_{i-1 / 2}^{l}\right.\right. \\
\left.\left.+\alpha_{i+3 / 2}^{l}\right)\right] .
\end{gathered}
$$

All above limiter functions are tested, and no qualitative difference is found for the present problem. Yee-Roe-Davis symmetric scheme with limiter Eq. (A16) is used in most computations as suggested by Ref. [25]. 
[1] D. F. Gaitan, L A. Crum, C. C. Church, and R. A. Roy, J. Acoust. Soc. Am. 91, 3166 (1992); E. B. Flint and K. S. Suslick, J. Am. Chem. Soc. 111, 6987 (1987); B. P. Barber and S. J. Putterman, Nature (London) 352, 318 (1991); B. P. Barber et al., J. Acoust. Soc. Am. 91, 3061 (1992); R. Hiller, S. J. Putterman, and B. P. Barber, Phys. Rev. Lett. 69, 1182 (1992); B. P. Barber and S. J. Putterman, ibid. 69, 3839 (1992); B. P. Barber et al., ibid. 72, 1380 (1994); B. P. Barber et al., Phys. Rep. 281, 65 (1997).

[2] P. Jarman, J. Acoust. Soc. Am. 32, 1459 (1960).

[3] C. C. Wu and P. H. Roberts, Phys. Rev. Lett. 70, 3424 (1993).

[4] W. Moss, D. B. Clarke, J. W. White, and D. A. Young, Phys. Fluids 6, 2979 (1994).

[5] L. Kondić, J. I. Gersten, and C. Yuan, Phys. Rev. E 52, 4976 (1995).

[6] M. P. Brenner, D. Lohse, D. Oxtoby, and T. F. Dupont, Phys. Rev. Lett. 76, 1158 (1996).

[7] I. Akhatov, N. Gumerov, C. D. Ohl, U. Parlitz, and W. Lauterborn, Phys. Rev. Lett. 78, 227 (1997).

[8] H. G. Flynn, J. Acoust. Soc. Am. 57, 1379 (1975).

[9] R. Hickling, J. Acoust. Soc. Am. 35, 967 (1963).

[10] M.-C. Chu and D. Leung, J. Phys.: Condens. Matter 9, 3387 (1997).

[11] L. A. Crum and S. Cordry, Proceedings of IUTAM Symposium on Bubble Dynamics and Interface Phenomena, edited by J. R. Blake and N. H. Thomas (Kluwer, Dordrecht, 1994).

[12] H. Y. Kwak and H. Yang, J. Phys. Soc. Jpn. 64, 1980 (1995).

[13] V. Kamath, A. Prosperetti, and F. N. Egolfopoulos, J. Acoust. Soc. Am. 94, 248 (1993).

[14] K. Yasui, J. Acoust. Soc. Am. 98, 2772 (1995).
[15] V. Q. Vuong and A. J. Szeri, Phys. Fluids 8, 2354 (1996).

[16] Lord Rayleigh, Philos. Mag. 34, 94 (1917); M. Plesset, J. Appl. Mech. 16, 277 (1949); B. Noltingk and E. Neppiras, Proc. Phys. Soc. London, Sect. B 63, 674 (1950); J. B. Keller and I. I. Kolodner, J. Appl. Phys. 27, 1152 (1956).

[17] R. Löfstedt, B. P. Barber, and S. J. Putterman, Phys. Fluids A 5, 2911 (1993).

[18] A. Prosperetti and A. Lezzi, J. Fluid Mech. 168, 457 (1986).

[19] J. B. Keller and M. Miksis, J. Acoust. Soc. Am. 68, 628 (1980).

[20] F. R. Gilmore, Caltech Hydrodynamics Laboratory Report No. 26-4 (1952).

[21] V. Kamath and A. Prosperetti, J. Acoust. Soc. Am. 85, 1538 (1987).

[22] M. S. Plesset and S. A. Zwick, J. Appl. Phys. 23, 95 (1952).

[23] C. E. Grosh and S. A. Orszag, J. Comput. Phys. 25, 273 (1977).

[24] S. Hilgenfeldt, D. Lohse, and M. P. Brenner, Phys. Fluids 8, 2808 (1996).

[25] H. C. Yee, NASA TM 101088, 1989 (unpublished).

[26] H. Schlichting, Boundary Layer Theory (McGraw-Hill Book Company, New York, 1968).

[27] Y. B. Zeldovich and Y. P. Raizer, Physics of Shock Waves and High-Temperature Hydrodynamic Phenomena, edited by W. D. Hayes and R. F. Probstein (Academic Press, New York, 1966), Vol. I.

[28] D. Lohse, M. P. Brenner, T. F. Dupont, S. Hilgenfeldt, and B. Johnston, Phys. Rev. Lett. 78, 1359 (1997).

[29] M. Vinokur, NASA CR 177512, 1988 (unpublished). 